\title{
Probing the circumnuclear absorbing medium of the buried AGN in NGC 1068 through NuSTAR observations
}

\author{
A. Zaino, ${ }^{1 \star}$ S. Bianchi, ${ }^{1}$ A. Marinucci, ${ }^{2}$ G. Matt, ${ }^{1}$ F. E. Bauer, ${ }^{3,4,5}$ \\ W. N. Brandt,,${ }^{6,8}$ P. Gandhi, ${ }^{9}$ M. Guainazzi, ${ }^{10}$ K. Iwasawa, ${ }^{11}$ S. Puccetti, ${ }^{2}$ \\ C. Ricci, ${ }^{12,13}$ D. J. Walton ${ }^{14}$ \\ ${ }^{1}$ Dipartimento di Matematica e Fisica, Università degli Studi Roma Tre, via della Vasca Navale 84, I-00146 Roma, Italy \\ ${ }^{2}$ Agenzia Spaziale Italiana (ASI) - Unità di Ricerca Scientifica, Via del Politecnico snc, I-00133 Roma, Italy \\ ${ }^{3}$ Instituto de Astrofísica and Centro de Astroingeniería, Facultad de Física, Pontificia Universidad Católica de Chile, Casilla 306, \\ Santiago 22, Chile \\ ${ }^{4}$ Millennium Institute of Astrophysics (MAS), Nuncio Monseñor Sótero Sanz 100, Providencia, Santiago, Chile \\ ${ }^{5}$ Space Science Institute, 4750 Walnut Street, Suite 205, Boulder, Colorado 80301, USA \\ ${ }^{6}$ Department of Astronomy and Astrophysics, The Pennsylvania State University, 525 Davey Laboratory, University Park, PA 16802, \\ $U S A$ \\ ${ }^{7}$ Institute for Gravitation and the Cosmos, The Pennsylvania State University, University Park, PA 16802, USA \\ ${ }^{8}$ Department of Physics, 104 Davey Laboratory, The Pennsylvania State University, University Park, PA 16802, USA \\ ${ }^{9}$ Department of Physics and Astronomy, University of Southampton, Highfield, Southampton, SO17 1BJ, UK \\ ${ }^{10}$ European Space Agency (ESA) - European Space Research and Technology Centre (ESTEC), Keplerlaan 1, NL-2201 AZ Noordwijk, \\ The Netherlands \\ ${ }^{11}$ ICREA and Institut de Ciències del Cosmos, Universitat de Barcelona, IEEC-UB, Martí i Franquès, 1, E-08028 Barcelona, Spain \\ ${ }^{12}$ Núcleo de Astronomía de la Facultad de Ingeniería, Universidad Diego Portales, Av. Ejército Libertador 441, Santiago, Chile \\ ${ }^{13}$ Kavli Institute for Astronomy and Astrophysics, Peking University, Beijing 100871, China \\ ${ }^{14}$ Institute of Astronomy, University of Cambridge, Madingley Road, Cambridge CB3 OHA, UK
}

Accepted 2020 January 10. Received 2020 January 9; in original form 2019 October 7.

\begin{abstract}
We present the results of the latest NuSTAR monitoring campaign of the Comptonthick Seyfert 2 galaxy NGC 1068, composed of four 50 ks observations performed between July 2017 and February 2018 to search for flux and spectral variability on timescales from 1 to 6 months. We detect one unveiling and one eclipsing event with timescales less than 27 and 91 days, respectively, ascribed to Compton-thick material with $N_{H}=(1.8 \pm 0.8) \times 10^{24} \mathrm{~cm}^{-2}$ and $N_{H} \geq(2.4 \pm 0.5) \times 10^{24} \mathrm{~cm}^{-2}$ moving across our line of sight. This gas is likely located in the innermost part of the torus or even further inward, thus providing further evidence of the clumpy structure of the circumnuclear matter in this source. Taking advantage of simultaneous Swift-XRT observations, we also detected a new flaring ULX, at a distance $d \sim 30$ " (i.e. $\sim 2 \mathrm{kpc}$ ) from the nuclear region of NGC 1068, with a peak X-ray intrinsic luminosity of $(3.0 \pm 0.4) \times 10^{40} \mathrm{erg} \mathrm{s}^{-1}$ in the $2-10 \mathrm{keV}$ band.
\end{abstract}

Key words: galaxies: active - galaxies: individual: NGC 1068 - galaxies: Seyfert X-rays: galaxies

\section{INTRODUCTION}

According to a widely accepted paradigm, AGN are powered by accretion of matter onto supermassive black holes (SMBHs) located in galactic centers (Lynden-Bell 1969). It is now well known that most AGN are "obscured" in the Xrays (e.g. Bianchi et al. 2012 and references therein; Ramos

^ E-mail: alessandra.zaino@uniroma3.it (AZ)
Almeida \& Ricci 2017) and at least part of their observed $\mathrm{X}$-ray variability is likely due to the variations of the circumnuclear medium surrounding the central engine (e.g. Risaliti et al. 2002; Yang et al. 2016). As stated by one popular AGN unification model (Antonucci 1993; Netzer 2015), this obscuring medium is optically thick, composed of dust and gas and arranged in an axisymmetric dusty structure with luminosity dependent dimensions of $0.1-10$ pc (i.e. the "torus"). Its column density is large enough to completely

(C) 2020 The Authors 
obscure the central source in some directions. The inclination of the line of sight with respect to the dusty torus is able to explain broadly the observational differences between Type 1 and Type 2 AGN, being the former observed at low angles with respect to the torus axis, while the latter are observed at high inclinations, with the nuclear region completely hidden by the torus itself. However, although X-ray studies have confirmed, to first order, the widely accepted unified scheme, the location, geometry and physical state of the absorbing material are still widely debated (Bianchi et al. 2012). In particular, the observation of short timescale variations ( days or even $\sim$ hours) in the absorption column density in several nearby bright sources, such as NGC 1365 (Risaliti et al. 2005; Rivers et al. 2015a), NGC 4151 (Puccetti et al. 2007), NGC 4388 (Elvis et al. 2004) and NGC 7582 (Bianchi et al. 2009; Rivers et al. 2015b), implies that the obscuring medium has a clumpy geometry, consisting of a series of discrete clouds likely at sub-pc scales. Although the density of this absorbing matter likely increases towards the equatorial plane (e.g. Nenkova et al. 2008a and 2008b), these variations are at odds with the classical torus geometry, which invokes a smooth distribution of dust and gas in a uniform toroidal structure (Pier \& Krolik 1992; Pier \& Krolik 1993; Fritz et al. 2006).

NGC $1068\left(D_{L}=14.4 \mathrm{Mpc}\right.$; Tully 1988) is one of the best known Seyfert 2 galaxies partly because the unification model was first proposed to explain the presence of broad optical lines in its polarized light (Antonucci \& Miller 1985). According to its classification, the nucleus of NGC 1068 is heavily obscured by dust. Near- and mid-infrared observations spatially resolved the dust structures within this galaxy, revealing a torus consistent with a two-component dust distribution. In particular, VLTI/VINCI observations for the first time favored a multi-component model for the torus intensity distribution. Taken into account also K-band speckle interferometry, these data showed that a part of the flux originates from scales clearly smaller than $\sim 0.4 \mathrm{pc}$, arising from substructures of the dusty torus or from the central accretion flow viewed through moderate extinction, and another part of the flux from larger scales of the order of $\sim 3 \mathrm{pc}$ (Wittkowski et al. 2004, and references therein). MIDI observations confirmed this scenario; in particular, Jaffe et al. (2004) modelled the observed spectra using two components with different size and temperature, each of which is a twodimensional Gaussian aligned with the axis parallel to the radio jet. They considered a central hot component $(\mathrm{T}>800$ $\mathrm{K}$ ) marginally resolved along the source axis and surrounded by a warm dust component $(\mathrm{T} \sim 320 \mathrm{~K})$ in a structure 2.1 parsec thick and 3.4 parsec in diameter. More recent MIDI observations allowed resolution of the nuclear mid-infrared emission from NGC 1068 in unprecedented detail, with a maximum resolution of 7 mas (i.e. $\sim 0.5 \mathrm{pc}$ at the distance of NGC 1068). In particular, Raban et al. (2009) found that the mid-infrared emission can be represented by two components, each with an elliptical Gaussian brightness distribution: a compact hot component $(\mathrm{T} \sim 800 \mathrm{~K}), 1.35$ parsec long and 0.45 parsec thick in FWHM (i.e. the inner funnel of the obscuring torus), tilted by $\sim 45^{\circ}$ with respect to the radio jet and with similar size and orientation to the observed water maser distribution (Gallimore et al. 2004), and a $3 \times 4$ pc warm component $(\mathrm{T} \sim 300 \mathrm{~K})$ marking the colder and extended part of the torus-like structure.
Recent ALMA observations have been able to resolve the molecular torus in NGC 1068 over spatial scales of $D=10-30 \mathrm{pc}$, demonstrating that there is radial density stratification as well as hints of counter-rotation and a high velocity outflow (Imanishi et al. 2018; García-Burillo et al. 2019; Impellizzeri et al. 2019). The observed physical parameters are significantly different depending on which line transition used to image the torus, highlighting its many faces. In particular, the $\mathrm{CO}(2-1), \mathrm{CO}(3-2)$ and $\mathrm{HCO}^{+}(4-3)$ maps provided a full-size of the torus $D_{C O(2-1)}=28 \pm 0.6$ pc, $D_{C O(3-2)}=26 \pm 0.6 \mathrm{pc}$ and $D_{\mathrm{HCO}^{+}(4-3)}=11 \pm 0.6 \mathrm{pc}$, respectively (García-Burillo et al. 2019).

From an X-ray point of view, the multi-epoch X-ray spectra of NGC 1068 were analysed by Bauer et al. (2015) using different observatories, including a $N u S T A R$ pointing in 2012 , and spanning a time period of $\sim 16$ years. The authors modeled the broadband emission of the source with a combination of a completely obscured transmitted power law $\left(\Gamma=2.10_{-0.07}^{+0.06}\right)$, scattering by both warm and cold reflectors, radiative recombination continuum and line emission, and off-nuclear point-source emission, being the latter due to the ULX population within the NGC 1068 field of view. In particular, the reflected emission was due to a multi-component reflector with three distinct column densities, in which the higher $N_{H}$ component $\left(N_{H, 1} \sim 10^{25} \mathrm{~cm}^{-2}\right)$ provides the bulk of the flux of the Compton hump, the lower $N_{H}$ component $\left(N_{H, 2}=(1.4 \pm 0.1) \times 10^{23} \mathrm{~cm}^{-2}\right)$ contributes primarily to the iron line emission and reproduces the curvature of the continuum around $10 \mathrm{keV}$, and a third reflector on more extended scales $(>140 \mathrm{pc}$ ) provides almost $30 \%$ of the neutral iron $\mathrm{K} \alpha$ line flux (see Appendix B and Bauer et al. 2015 for further details).

NGC 1068 was observed again in 2014 and in 2015 with a joint XMM-Newton and NuSTAR monitoring campaign during which a transient excess above $20 \mathrm{keV}$ was observed and ascribed to a Compton-thick unveiling event in which material with $N_{H} \geq 2.5 \times 10^{24} \mathrm{~cm}^{-2}$ moved temporarily out of our line of sight (Marinucci et al. 2016), allowing the intrinsic radiation to pierce through the circumnuclear medium. VISIR and MIDI observations performed before and immediately after the X-ray variations showed constant behavior in the infrared emission of the nuclear region of NGC 1068, confirming the hypothesis that the observed change in the $\mathrm{X}$-ray regime was not due to an intrinsic change in the luminosity of the central accretion disk, but to escaping emission through the patchy torus clouds (López-Gonzaga et al. 2017). However, due to the large separation of the NuSTAR observations ( $\sim 6$ months), only an upper limit of $\sim 2$ pc could be given on the location of such a variable absorber, which is consistent both with the BLR and the torus. In the latter case, the change in the absorbing column density along the line of sight required to explain the X-ray variability of the AGN in NGC 1068 could be associated with the small-scale structure of the molecular torus imaged by ALMA (GarcíaBurillo et al. 2019).

In this paper, we present results from the latest $\mathrm{NuS}$ $T A R$ monitoring campaign of NGC 1068 performed between July 2017 and February 2018 to sample variability timescales from 1 to 6 months. The aim was to search for flux and spectral variability on timescales not covered during the previous monitoring campaign and providing tighter constraints on the circumnuclear absorbing Compton-thick material, its 
Table 1. NuSTAR observation log for NGC 1068.

\begin{tabular}{|c|c|c|c|c|c|c|c|}
\hline & obsID & Start time & Stop time & Detector & $\begin{array}{l}\text { Net exposure } \\
\text { time }{ }^{a}[\mathrm{ks}]\end{array}$ & $\begin{array}{l}\text { Net count rate } \\
3-5.5 \mathrm{keV}\end{array}$ & $\begin{array}{c}b\left[\text { counts s }{ }^{-1}\right] \\
20-79 \mathrm{keV}\end{array}$ \\
\hline \multirow[t]{2}{*}{ OBS1 } & 60302003002 & 2017-07-31 00:16:09 & 2017-08-01 03:26:09 & FPMA & 50.0 & $0.0246 \pm 0.0007$ & $0.0289 \pm 0.0009$ \\
\hline & & & & FPMB & 49.8 & $0.0240 \pm 0.0007$ & $0.0262 \pm 0.0008$ \\
\hline \multirow[t]{2}{*}{ OBS2 } & 60302003004 & 2017-08-27 20:51:09 & 2017-08-29 03:36:09 & FPMA & 52.5 & $0.0253 \pm 0.0007$ & $0.0332 \pm 0.0009$ \\
\hline & & & & FPMB & 52.4 & $0.0256 \pm 0.0007$ & $0.0309 \pm 0.0009$ \\
\hline \multirow[t]{2}{*}{ OBS3 } & 60302003006 & 2017-11-06 03:31:09 & 2017-11-07 06:31:09 & FPMA & 49.7 & $0.0254 \pm 0.0007$ & $0.0301 \pm 0.0009$ \\
\hline & & & & FPMB & 49.5 & $0.0236 \pm 0.0007$ & $0.0281 \pm 0.0008$ \\
\hline \multirow[t]{2}{*}{ OBS4 } & 60302003008 & 2018-02-05 05:26:09 & 2018-02-06 11:36:09 & FPMA & 54.6 & $0.0313 \pm 0.0008$ & $0.0276 \pm 0.0008$ \\
\hline & & & & FPMB & 54.5 & $0.0299 \pm 0.0008$ & $0.0261 \pm 0.0008$ \\
\hline
\end{tabular}

Notes. $^{a}$ Net exposure time, after screening was applied on the data. ${ }^{b}$ Net source count rate after screening and background subtraction, as observed in the $3-5.5 \mathrm{keV}$ and 20-79 keV energy ranges.

physical properties, and its distance from the illuminating source.

The paper is organized as follows. In Section 2 we discuss the X-ray observations and data reduction, with Sections 2.1 and 2.2 devoted to NuSTAR and Swift-XRT data, respectively. In Section 3 we describe the X-ray spectral analysis, while in Section 4 we discuss our findings, comparing them with the previous X-ray results and, finally, in Section 5 we summarize our work. The detection of a new ULX is discussed in Appendix A, while a detailed description of the best-fit model adopted by Bauer et al. (2015), which is the basis of our modelling, is in Appendix B. Finally, Appendix $\mathrm{C}$ is devoted to calibration uncertainties.

Throughout the paper, we assumed a flat $\Lambda \mathrm{CDM}$ cosmology with $\mathrm{H}_{0}=70 \mathrm{~km} \mathrm{~s}^{-1} \mathrm{Mpc}^{-1}, \Omega_{\Lambda}=0.73$ and $\Omega_{\mathrm{m}}=0.27$, i.e. the default ones in XSPEC 12.10.1 (Arnaud 1996).

\section{OBSERVATIONS AND DATA REDUCTION}

\section{$2.1 \quad N u S T A R$}

NGC 1068 was observed by NuSTAR (Harrison et al. 2013) with its two co-aligned X-ray telescopes, with corresponding Focal Plane Modules A (FPMA) and B (FPMB), during a monitoring campaign composed of four observations of about 50 ks each, performed between July 2017 and February 2018 (see Table 1).

The Level 1 data products were processed using the NuSTAR Data Analysis Software (NuSTARDAS) package (version 1.8.0). Event files (Level 2 data products) were extracted using the nupipeline task, adopting standard filtering criteria and the latest calibration files available in the NuSTAR calibration database (CALDB 20180126). The source spectra were extracted from circular regions of radius 50 arcsec, corresponding to an encircled energy fraction (EEF) of about $70 \%$, from both FPMA and FPMB. The same radius was used to extract background spectra, selecting a region on the same chip, uncontaminated by source photons or background sources. The net exposure times and the total count rates after this process are reported in Table 1 for each spectrum and for both FPMA and FPMB.

Finally, the two NuSTAR spectra from each observation were binned in order not to oversample the instrumental energy resolution by a factor larger than 4 and to have a signalto-noise ratio (SNR) greater than 7 in each backgroundsubtracted spectral channel. This ensures the applicability
Table 2. Swift-XRT observation log for NGC 1068.

\begin{tabular}{cccc}
\hline obsID & Start time & $\begin{array}{c}\text { Exposure time } \\
{[\mathrm{ks}]}\end{array}$ & \\
\hline 0088104005 & $2017-11-06 \quad 08: 40: 57$ & 2.0 & $(1)$ \\
0088104006 & $2018-02-05 \quad 05: 27: 57$ & 2.1 & $(2)$ \\
0088104007 & $2018-06-15 \quad 16: 13: 48$ & 1.6 & $(3)$ \\
\hline
\end{tabular}

Notes. (1) Simultaneous to OBS3. (2) Simultaneous to OBS4

(3) $\mathrm{ToO}$ observation.

of the $\chi^{2}$ statistic to evaluate the quality of spectral fitting, avoiding a dramatic oversampling due to the high flux of the source. The same energy bins were used for both FPMA and FPMB.

\subsection{Swift}

In our work we also analyzed two Swift-XRT archival observations performed simultaneously with NuSTAR in November 2017 and February 2018 and a ToO observation requested in June 2018 (see Table 2).

To analyze the XRT observation taken in February 2018 , we extracted a source spectrum from a circular region with a 20 arcsec radius centered on the ULX (see Appendix A for further details), ensuring that the nuclear emission of NGC 1068 is excluded. A background spectrum was also extracted from a source-free circular region with a 60 arcsec radius. We used identical extraction regions for the other two XRT spectra (November 2017 and June 2018), generating spectra with XSELECT, effective area files with XRTMKARF, and using the redistribution matrix file SWXPC0TO12s6_20130101v014.RMF. Finally, all the XRT spectra were rebinned in order to have at least 15 counts per energy bin.

\section{X-RAY SPECTRAL ANALYSIS}

The X-ray spectral analysis of NGC 1068 was performed using XSPEC 12.10.1 (Arnaud 1996) and the $\chi^{2}$ statistic, apart from the ULX analysis (see Appendix A), where the C-statistic (Cash 1976) was used. The photoelectric cross sections for all absorption components are those from Verner et al. (1996), while the element abundance pattern is from Wilms et al. (2000) and the metal abundance is fixed to solar. Unless stated otherwise, errors correspond to 


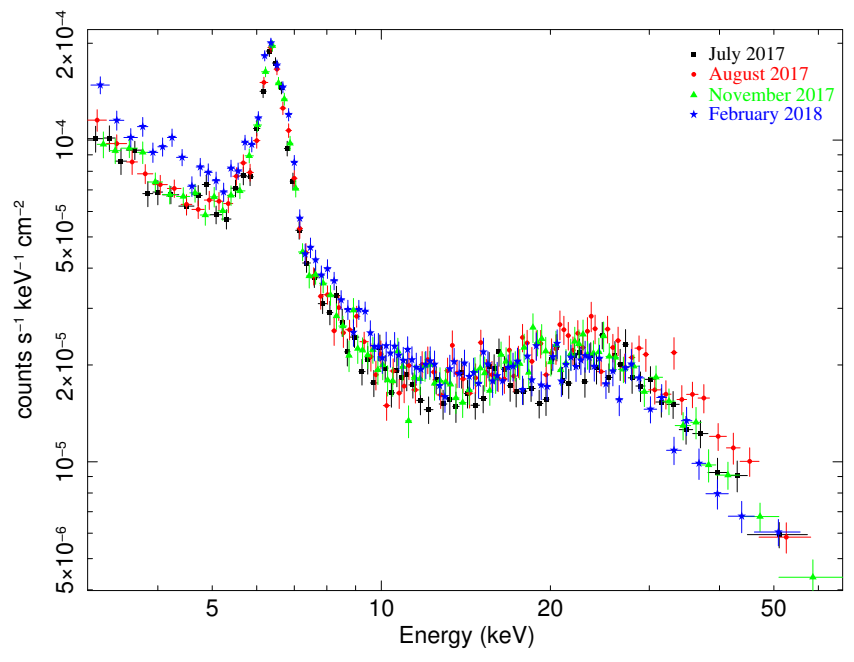

Figure 1. NuSTAR monitoring spectra taken in July 2017 (black squares), August 2017 (red circles), November 2017 (green triangles) and February 2018 (blue stars). FPMA and FPMB data of each observation are grouped together for clarity purposes only.

the 90 per cent confidence level for one interesting parameter $\left(\Delta \chi^{2}=2.706\right)$. The four NuSTAR monitoring spectra are shown in Figure 1.

Focusing on the soft X-ray band below $5.5 \mathrm{keV}$, we can observe an excellent agreement of the data taken in July, August and November 2017 (see black, red and green symbols in Figure 1, respectively). However, an unexpected increase of the flux by $25 \pm 2 \%$ was observed in February 2018 with respect to the previous observations (see blue circles in Figure 1 and the count-rates in Table 1). Swift-XRT observations reported in Table 2 confirmed the appearance of a transient source at a distance of $\sim 2 \mathrm{kpc}$ (i.e. $\sim 30$ arcsec) from the nucleus of NGC 1068 as the origin of this excess (see Appendix A for further details).

Considering the whole NuSTAR band (3-79 keV), we find that the iron line emission does not vary significantly during the whole monitoring (see Figure 1); however, the count-rate above $20 \mathrm{keV}$ clearly varies by up to $\sim 20 \%$ between the observations (see Table 1). This behavior is similar to that observed three years previously; in particular, NGC 1068 was caught in a higher flux state in August 2014 and 2017 and in a lower one in February 2015 and 2018, as reported in Table 3. Additionally, we find excellent overlap of the spectral shapes in the high and low states during the two NuSTAR campaigns (see Figure 2). Both these findings suggest that we are observing eclipsing/unveiling events affecting only the spectrum above $10 \mathrm{keV}$, as found in Marinucci et al. (2016). The benefit of the current NuSTAR monitoring campaign is that we have two additional observations in July and November 2017 (see Table 1) that enable us to more accurately probe the variability of NGC 1068 .

To model our data set, we adopted the best-fit model discussed in Bauer et al. 2015 (see Appendix B for a detailed description of the model), with an additional cut-off power-law component to account for the ULX contribution in OBS4 (Model A in Table 4), whose spectral analysis is described in Appendix A. Due to the fact that ULXs break to very steep spectra above $\sim 10 \mathrm{keV}$ (e.g. Stobbart et al.
Table 3. Comparison between the higher and the lower flux states of NGC 1068 during 2014-2015 and 2017-2018 NuSTAR monitoring.

\begin{tabular}{|c|c|c|c|}
\hline \multicolumn{2}{|c|}{ High state } & \multicolumn{2}{|c|}{ Low state } \\
\hline Date & Count rate ${ }^{(1)}$ & Date & Count rate ${ }^{(1)}$ \\
\hline \multicolumn{4}{|c|}{ FPMA } \\
\hline Aug 2014 & $0.032 \pm 0.001$ & Feb 2015 & $0.026 \pm 0.001$ \\
\hline Aug 2017 & $0.033 \pm 0.001$ & Feb 2018 & $0.028 \pm 0.001$ \\
\hline \multicolumn{4}{|c|}{ FPMB } \\
\hline Aug 2014 & $0.031 \pm 0.001$ & Feb 2015 & $0.023 \pm 0.001$ \\
\hline Aug 2017 & $0.031 \pm 0.001$ & Feb 2018 & $0.026 \pm 0.001$ \\
\hline
\end{tabular}

2006, Gladstone et al. 2009, Pintore et al. 2017, Walton et al. 2018), we expect that this new source should have a negligible effect on the high-energy data from the nucleus. In our configuration, the reflected emission is reproduced by three distinct reflectors $\left(\theta_{1}=90^{\circ}, N_{H, 1} \geq 9.7 \times 10^{24} \mathrm{~cm}^{-2} ; \theta_{2}=0^{\circ}\right.$, $N_{H, 2}=(1.4 \pm 0.1) \times 10^{23} \mathrm{~cm}^{-2} ; \theta_{3}=0^{\circ}, N_{H, 3}=\left(5.0_{-1.9}^{+4.2}\right) \times 10^{24}$ $\mathrm{cm}^{-2}$ ) modeled with MYTORUS tables in a decoupled configuration (Yaqoob 2012), where the normalizations for the different angles vary independently, while the continuum and line components of a given angle are fixed, corresponding to a patchy torus distribution. Applying this model to our data set, the fit was not good $\left(\chi_{r}^{2}=1.63\right)$, mainly due to significant residuals at $\sim 6 \mathrm{keV}$ (see panel (a) in Figure 3).

Taking advantage of previous NuSTAR observations, some of which were performed simultaneously with $X M M$ Newton, we argue that the significant residuals observed in Model A have no astrophysical origin, but are spurious calibration features (see Appendix C for a detailed analysis). We modeled this component out of the residuals adopting an additional emission line (i.e. a gauss component in XSPEC), whose parameters are reported in Table 4 (Model B), obtaining a significant improvement of the fit $\left(\chi_{r}^{2}=1.18-\right.$ see panel (b) in Figure 3). We stress that this further component does not affect our conclusions.

Moreover, to better compare our findings to those obtained from the previous NuSTAR analyses, we decided to leave the column densities and normalizations of the reflection parameters free to vary (Model C $-\chi_{r}^{2}=1.08-$ panel (c) in Figure 3). This allowed us to check the good agreement between our values and those observed by Bauer et al. (2015) for the 2012 epoch and Marinucci et al. (2016) for the 2014-2015 epochs, as reported in Table 5. Since the highest column density reflector located within 2 arcsec of the nuclear region and providing the bulk of the flux of the Compton hump (i.e. $N_{H, 1}$ ) was not constrained, we fixed its value to the best-fit one (i.e. $10^{25} \mathrm{~cm}^{-2}$ ).

Then, due to the complexity of the model used to reproduce our data set (see Appendix B for a detailed description), we decided to follow the same approach used by Marinucci et al. (2016) in analyzing the 2014-2015 NuSTAR campaign. Therefore, we fixed the column densities and the normalizations of the three reflectors to the best-fit values previously found, leaving only the flux of the primary component and the column density along the line of sight free to vary (Model D). We obtained an improvement of the fit $\left(\chi_{r}^{2}=1.07-\right.$ panel $(\mathrm{d})$ in Figure 3$)$, pointing out a varia- 
Table 4. Summary of the six models discussed in the text and differences with respect to the Bauer model summarized in Appendix B.

\begin{tabular}{cccc}
\hline \multicolumn{4}{c}{ Model A: Bauer model + ULX contribution in OBS4 } \\
\hline$\Gamma_{\mathrm{ULX}}$ & $\mathrm{E}_{\mathrm{c}}[\mathrm{keV}]$ & norm $\mathrm{ULX}\left[\mathrm{ph} \mathrm{cm}^{-2} \mathrm{~s}^{-1} \mathrm{keV}^{-1}\right]$ & $\chi^{2} / \mathrm{dof}$ \\
$1.5 \pm 0.4$ & $\geq 5$ & $\left(2.6_{-0.6}^{+0.7}\right) \times 10^{-4}$ & $1186 / 730$
\end{tabular}

\begin{tabular}{cccc}
\hline \multicolumn{4}{c}{ Model B: Model A + gauss component at $\sim 6 \mathrm{keV}$} \\
\hline & $\begin{array}{c}\text { Egauss }(\text { FPMA/FPMB) } \\
{[\mathrm{keV}]}\end{array}$ & $\begin{array}{c}\text { norm gauss }(\text { FPMA/FPMB }) \\
{\left[\mathrm{ph} \mathrm{cm}^{-2} \mathrm{~s}^{-1} \mathrm{keV}^{-1}\right]}\end{array}$ & $\chi^{2} /$ dof \\
OBS1 & $6.1 \pm 0.1 / 6.1 \pm 0.1$ & $1.7 \pm 0.5 / 2.5 \pm 0.5$ & \\
OBS2 & $6.1 \pm 0.1 / 6.1 \pm 0.1$ & $1.3 \pm 0.5 / 2.3 \pm 0.5$ & $840 / 714$ \\
OBS3 & $6.0 \pm 0.1 / 6.1 \pm 0.1$ & $2.1 \pm 0.5 / 2.2 \pm 0.5$ & \\
OBS4 & $5.9 \pm 0.1 / 5.9 \pm 0.1$ & $1.7 \pm 0.4 / 1.9 \pm 0.5$ &
\end{tabular}

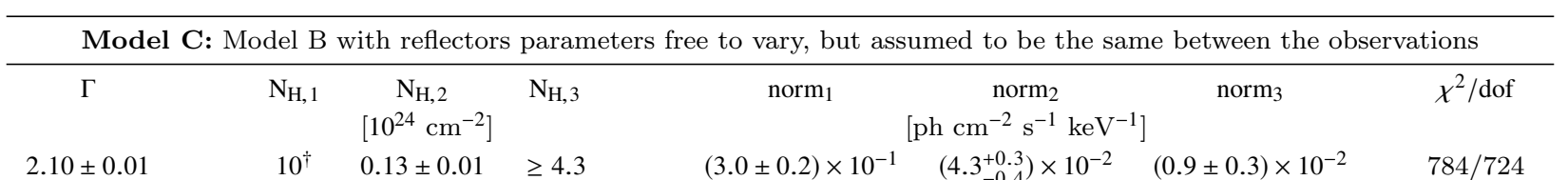

$10^{\dagger} \quad 0.13 \pm 0.01 \geq 4.3$

$(3.0 \pm 0.2) \times 10^{-1} \quad\left(4.3_{-0.4}^{+0.3}\right) \times 10^{-2}$

$(0.9 \pm 0.3) \times 10^{-2}$

$784 / 724$

\begin{tabular}{|c|c|c|c|}
\hline \multicolumn{4}{|c|}{ Model D: Model C with only $N_{\boldsymbol{H}}$ along the l.o.s. and intrinsic flux free to vary } \\
\hline & $\mathrm{N}_{\mathrm{H}}^{1 . \text { o.s. }}\left[10^{24} \mathrm{~cm}^{-2}\right]$ & norm $_{\text {intr }}\left[\mathrm{ph} \mathrm{cm}^{-2} \mathrm{~s}^{-1} \mathrm{keV}^{-1}\right]$ & $\chi^{2} /$ dof \\
\hline OBS1 & $\geq 7.3$ & $5.0_{-4.6}^{+2.1}$ & \\
\hline OBS2 & $6.3_{-1.0}^{+1.2}$ & $0.9_{-0.5}^{+1.0}$ & $784 / 730$ \\
\hline OBS3 & $4.6_{-1.2}^{+1.4}$ & $0.12_{-0.08}^{+0.28}$ & \\
\hline OBS4 & $2.4^{\dagger}$ & $\leq 1.2$ & \\
\hline \multicolumn{4}{|c|}{ Model E: Model D with the same intrinsic luminosity during the monitoring } \\
\hline & $\mathrm{N}_{\mathrm{H}}^{\text {l.o.s. }}\left[10^{24} \mathrm{~cm}^{-2}\right]$ & norm $_{\text {intr }}\left[\mathrm{ph} \mathrm{cm}^{-2} \mathrm{~s}^{-1} \mathrm{keV}^{-1}\right]$ & $\chi^{2} /$ dof \\
\hline OBS1 & $7.4_{-1.0}^{+1.1}$ & \multirow{4}{*}{$0.5_{-0.2}^{+0.5}$} & \multirow{4}{*}{$793 / 733$} \\
\hline OBS2 & $5.6_{-0.8}^{+0.9}$ & & \\
\hline OBS3 & $6.2_{-0.8}^{+1.0}$ & & \\
\hline OBS4 & $\geq 7.8$ & & \\
\hline \multicolumn{4}{|c|}{ Model F: Model D with the same absorbing column density during the monitoring } \\
\hline & $\mathrm{N}_{\mathrm{H}}^{1.0 . s .}\left[10^{24} \mathrm{~cm}^{-2}\right]$ & norm $_{\text {intr }}\left[\mathrm{ph} \mathrm{cm}^{-2} \mathrm{~s}^{-1} \mathrm{keV}^{-1}\right]$ & $\chi^{2} /$ dof \\
\hline OBS1 & \multirow{4}{*}{$5.9_{-0.8}^{+1.0}$} & $0.15_{-0.10}^{+0.25}$ & \multirow{4}{*}{$793 / 733$} \\
\hline OBS2 & & $0.7_{-0.4}^{+0.8}$ & \\
\hline OBS3 & & $0.38_{-0.20}^{+0.4}$ & \\
\hline OBS4 & & $\leq 0.14$ & \\
\hline
\end{tabular}

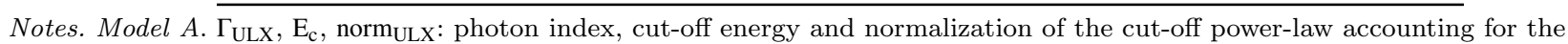
contribution of the ULX discussed in Appendix A. Model B. Egauss and norm gauss: energy and normalization of the phenomenological line due to calibration issues modeled in XSPEC with $\sigma=0$ and discussed in Appendix C. Model C. $\Gamma$ : photon index of the primary continuum; $\mathrm{N}_{\mathrm{H}, 1}, \mathrm{~N}_{\mathrm{H}, 2}, \mathrm{~N}_{\mathrm{H}, 3}$ and norm 1 , norm 2 , norm 3 : column densities and normalizations of the three reflectors, respectively. Model

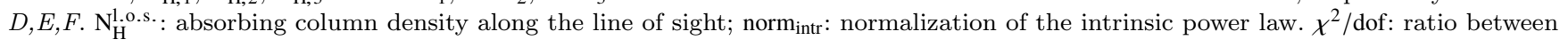
$\chi^{2}$ and the degrees of freedom of the model. ${ }^{\dagger}$ Unconstrained value. Errors correspond to the 90 per cent confidence level for one interesting parameter. In all models, ULX parameters are fixed to their best-fit values, while the column density along the line of sight and the intrinsic luminosity of the source are left free to vary (for clarity purposes, these values are not reported here for Model A,B,C). All the other parameters not shown in this table are fixed to the best-fit values reported in Bauer et al. (2015).

Table 5. Reflectors parameters modeled through MYTORUS tables (Murphy \& Yaqoob 2009).

\begin{tabular}{|c|c|c|c|c|c|c|c|}
\hline & $\Gamma^{(1)}$ & $\mathrm{N}_{\mathrm{H}, 1}$ & norm $_{1}(3)$ & $\mathrm{N}_{\mathrm{H}, 2}$ & $\operatorname{norm}_{2}(3)$ & $\mathrm{N}_{\mathrm{H}, 3}$ & norm $_{3}(3)$ \\
\hline Bauer (2015) & $2.10_{-0.07}^{+0.06}$ & $\geq 9.7$ & $(3.0 \pm 0.5) \times 10^{-1}$ & $0.14 \pm 0.01$ & $\left(3.6_{-0.2}^{+0.3}\right) \times 10^{-2}$ & $5.0_{-1.9}^{+4.2}$ & $(1.0 \pm 0.2) \times 10^{-2}$ \\
\hline
\end{tabular}

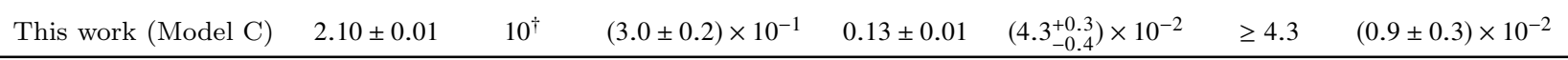
Notes. (1) Photon index. (2) Column density of the reflector, in units of $10^{24} \mathrm{~cm}^{-2}$. (3) Normalization of the reflected emission, in units of ph $\mathrm{cm}^{-2} \mathrm{~s}^{-1} \mathrm{keV}^{-1}$. ${ }^{\dagger}$ Fixed value. 

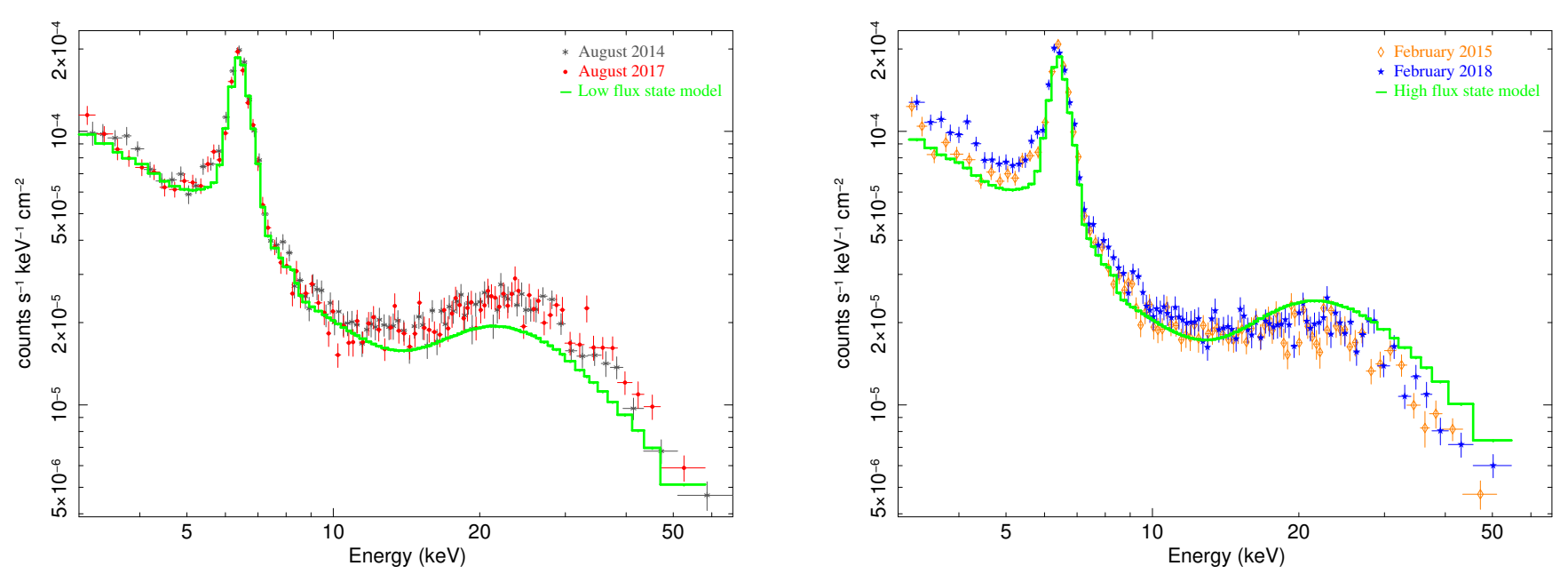

Figure 2. NuSTAR observations of NGC 1068 during the last two NuSTAR monitoring campaigns. Left panel. Comparison between OBSID 60002033002 (August 2014; grey asterisks) and OBS2 (August 2017; red circles), catching the source in a high flux state. Right panel. Comparison between OBSID 60002033004 (February 2015; orange diamonds) and OBS4 (February 2018; blue stars), observing the source in a low flux state. In both panels, the green solid line represents the modelling of the opposite flux state, and FPMA and FPMB data of each observation are grouped together for clarity purposes only. The disagreement between blue data and the high flux model below $5.5 \mathrm{keV}$ is due to the appearence of the ULX in February 2018. We refer to the text for further details.

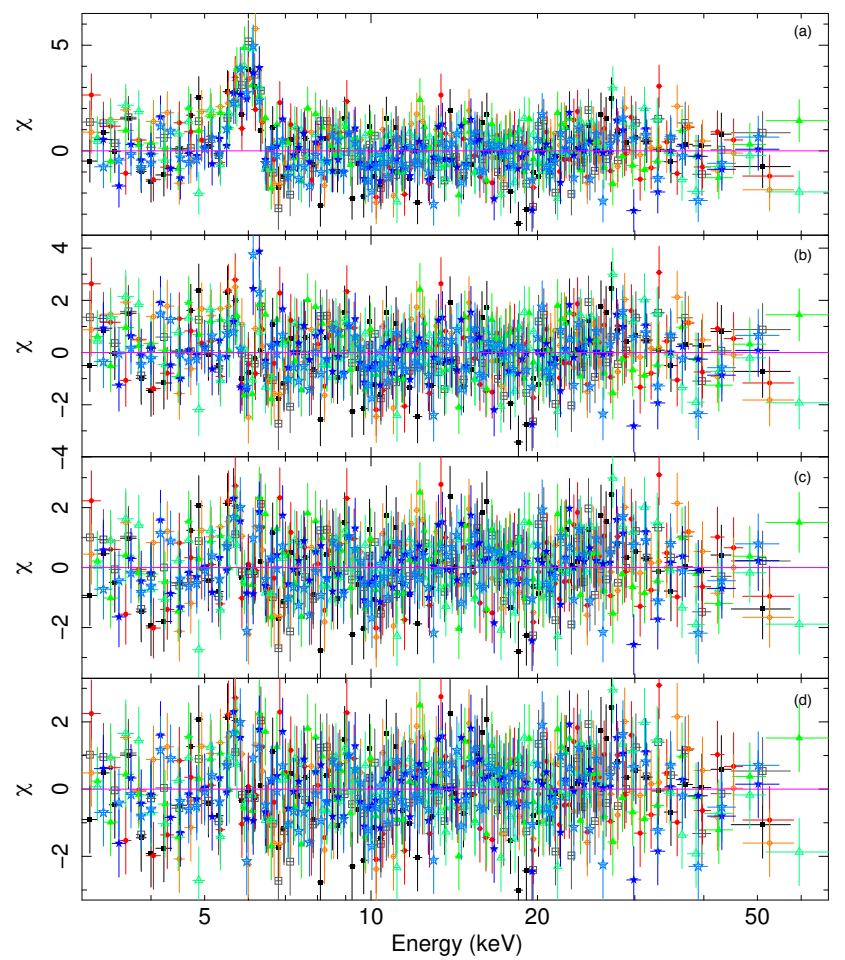

Figure 3. From top to bottom. Residuals with respect to Model $\mathrm{A}, \mathrm{B}, \mathrm{C}$ and $\mathrm{D}$ (solid magenta line in the four panels) plotted in terms of sigmas. OBS1, OBS2, OBS3 and OBS4 are shown in black and grey squares, red and orange circles, green and dark green triangles and blue and light blue stars, while filled and empty symbols represent FPMA and FPMB data, respectively.

tion in $N_{H}$ between the observations. However, the column density along the line of sight in OBS4 is unconstrained and a significant spread in the intrinsic emission of the AGN is clearly visible during the monitoring (see Figure 4 and
Model D in Table 4), although the large errors make the normalizations of the four observations consistent with each other.

For this reason, we tied together the normalizations of the intrinsic power-law during the whole monitoring, leaving only the column density along the line of sight free to vary (Model E - see Figure 5). Despite the $\chi_{r}^{2}$ being unchanged with respect to Model $\mathrm{D}$, this modelling allowed us to avoid the degeneracy between variations in the absorbing column density and the intrinsic emission of the source resulting in better constraints on the column density in all the observations (see Model E in Table 4) and reproducing well the behavior already shown in Figure 2. Using this parametrization, we obtained an intrinsic unabsorbed 2-10 keV luminosity $L_{2-10}=\left(3.5_{-1.8}^{+3.6}\right) \times 10^{43} \mathrm{erg} \mathrm{s}^{-1}$ at the distance of NGC 1068, in agreement with Bauer et al. (2015) and Marinucci et al. (2016), and fully consistent with the one inferred using the mid-IR (Gandhi et al. 2009) and [OIII] (Lamastra et al. 2009) observed luminosities.

On the other hand, the degeneracy between $N_{H}$ and the intrinsic emission of the source could be also avoided assuming a uniform absorbing column density with an intrinsic variability in the accretion rate. Testing this scenario (Model $\mathrm{F}$ in Table 4), we obtained a fit equivalent to the previous one from a statistical point of view $\left(\chi_{r}^{2}=1.08\right)$, with an absorbing column density along the line of sight $N_{H}=\left(5.9_{-0.8}^{+1.0}\right) \times 10^{24} \mathrm{~cm}^{-2}$, fully consistent with the values obtained in Model D only for two observations (OBS2 and OBS3). Furthermore, a variation between the normalizations of the intrinsic power-law in OBS3 and OBS4 is clearly visible. This corresponds to a change in the X-ray luminosity in the $2-10 \mathrm{keV}$ band $\Delta L_{2-10}=\left(1.8_{-1.4}^{+3.3} \times 10^{43}\right) \mathrm{erg} \mathrm{s}^{-1}$, suggesting a decrease in the normalized accretion rate of the source (i.e. $\mathrm{L}_{\mathrm{bol}} / \mathrm{L}_{\mathrm{Edd}}$ ) from 0.47 to 0.12 in a 3 -month timescale.

However, due to the fact that the X-ray luminosity we obtain in the low-flux state of NGC 1068 (which characterises most of the observations) using Model F (i.e. 

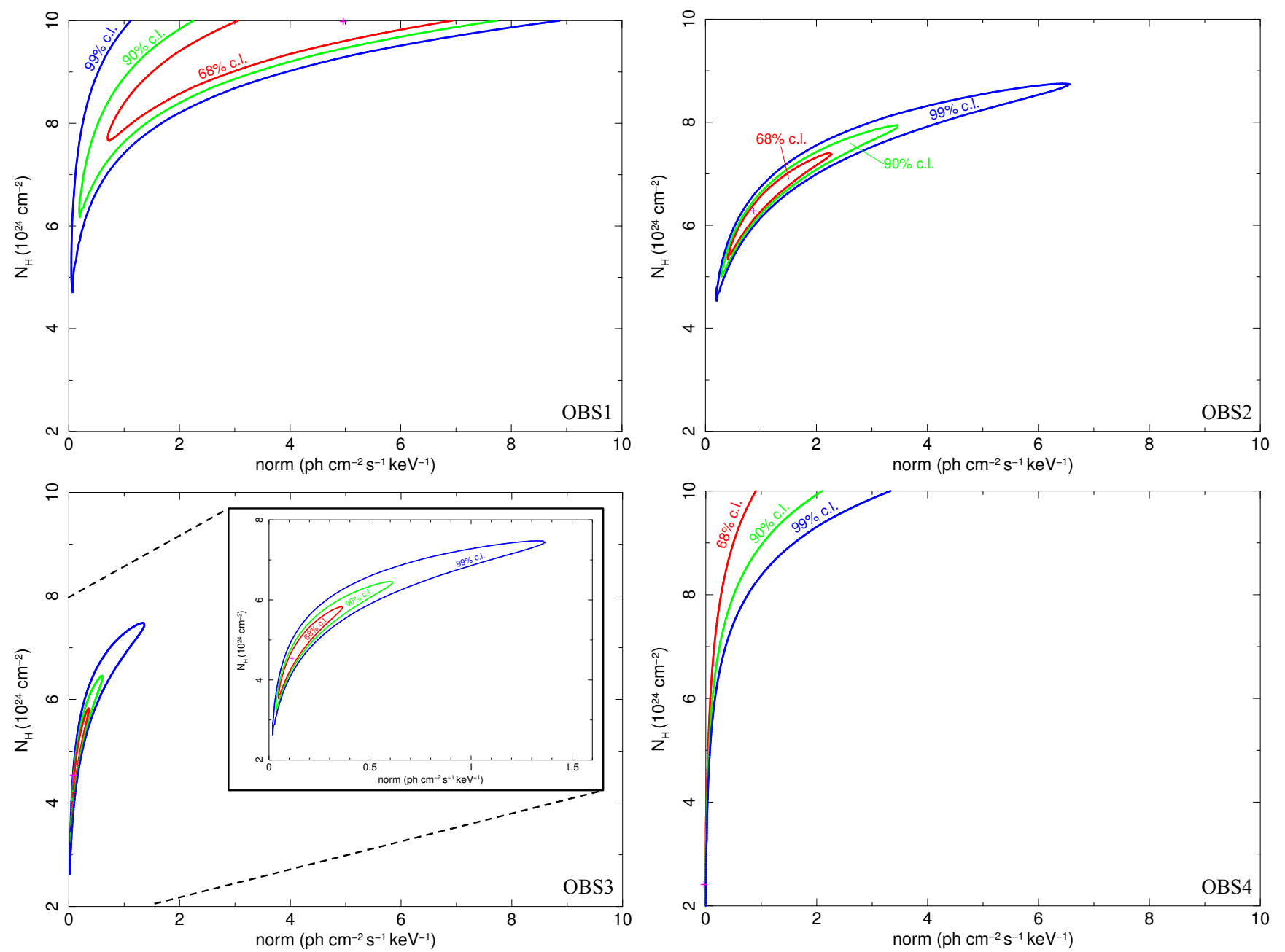

Figure 4. Confidence contours between the obscuring column density along the line of sight and the normalization of the primary power-law component for OBS1 (top left), OBS2 (top right), OBS3 (bottom left - a zoom is shown within the black box), and OBS4 (bottom right), when Model D is assumed. A variation in $N_{\boldsymbol{H}}$ is clearly visible between the observations, even if the absorbing column density is unconstrained in OBS4, while the normalizations are consistent with each other despite a large spread in their values. Red, green and blue contours indicate 68 per cent, 90 per cent and 99 per cent confidence levels, while the magenta cross indicates the best-fit values.

$L_{2-10} \leq 9.8 \times 10^{42} \mathrm{erg} \mathrm{s}^{-1}$ ) is much less than the one expected from other wavelengths, while the constant $L_{X}$ - multiple $N_{H}$ scenario provides an X-ray luminosity fully consistent with the one inferred using the mid-IR and [OIII] observed luminosities, we adopted Model E as our best-fit model.

\section{DISCUSSION}

Supposing that the intrinsic luminosity of the source did not vary during the whole monitoring, we attribute the spectral differences observed above $20 \mathrm{keV}$ to X-ray emission piercing through a patchy dusty region. We observe the same column density variability on 6 -month timescales already found by Marinucci et al. (2016) during the previous NuSTAR monitoring campaign (see red and blue data points with respect to grey and orange ones in Figure 6), suggesting the presence of a variable absorber on parsec-scale distance. However, the observational strategy of our monitoring campaign allowed us to probe shorter timescales with the aim of better con- straining the location of the circumnuclear absorbing matter in NGC 1068.

According to our best-fit model, the values of the column density along the line of sight with their 1-sigma errors are $N_{H}=(7.4 \pm 0.6) \times 10^{24} \mathrm{~cm}^{-2}, N_{H}=(5.6 \pm 0.5) \times 10^{24}$ $\mathrm{cm}^{-2}, N_{H}=(6.2 \pm 0.5) \times 10^{24} \mathrm{~cm}^{-2}$ and $N_{H} \geq 8.6 \times 10^{24} \mathrm{~cm}^{-2}$ (corresponding to $N_{H} \geq 7.8 \times 10^{24} \mathrm{~cm}^{-2}$ at the 90 per cent confidence level, as reported in Table 4) for OBS1, OBS2, OBS3 and OBS4, respectively (see Figure 6). Thus, assuming the same intrinsic X-ray luminosity during the whole monitoring, we could clearly identify one unveiling and one eclipsing event: the first between July and August 2017 and the second between November 2017 and February 2018 with timescales lower than 27 days and 91 days, respectively. These events were ascribed to Compton-thick material with $N_{H}=(1.8 \pm 0.8) \times 10^{24} \mathrm{~cm}^{-2}$ and $N_{H} \geq(2.4 \pm 0.5) \times 10^{24}$ $\mathrm{cm}^{-2}$, respectively, which moved temporarily across our line of sight, allowing or preventing the intrinsic nuclear radiation to pierce through the circumnuclear absorbing medium.

Considering a scenario in which the obscuring mate- 


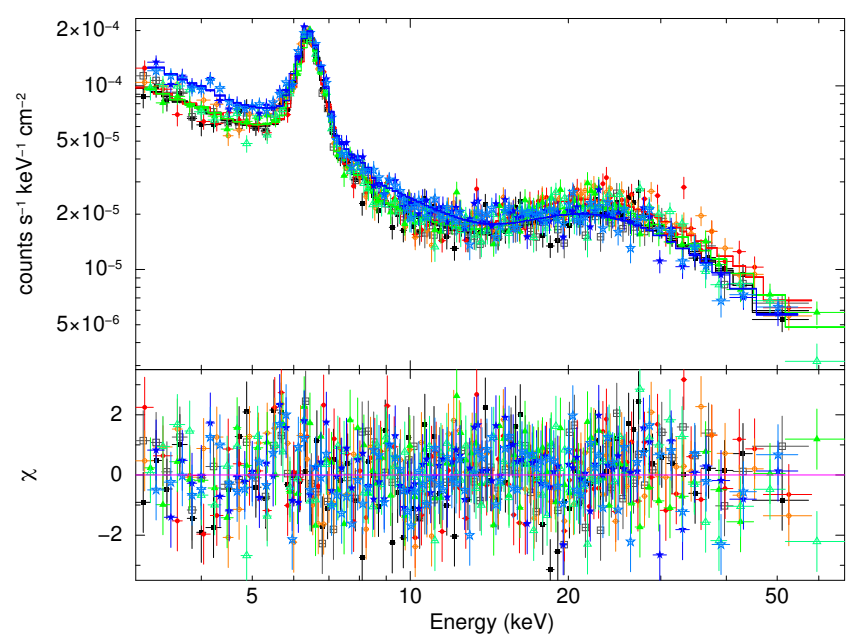

Figure 5. NUSTAR monitoring spectra (top panel) and residuals plotted in terms of sigmas (bottom panel) with respect to the best-fit model (Model E). Both the color code and the symbols are the same as in Figure 3.

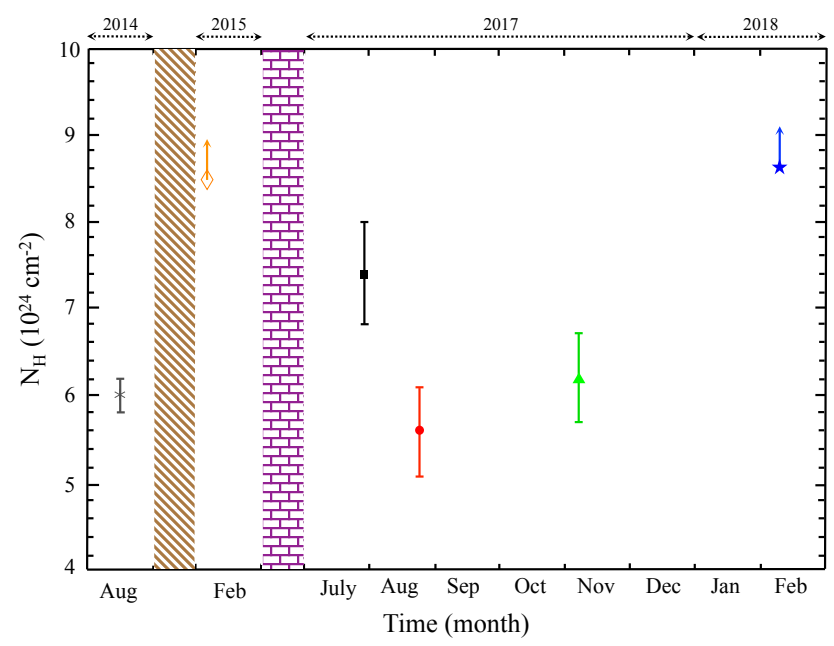

Figure 6. Values of the obscuring column density along the line of sight for the four 2017-2018 NuSTAR monitoring observations (black square, red circle, green triangle and blue star), when Model E is adopted. Old 2014-2015 measurements (grey asterisk and orange diamond) reproducing the same intrinsic luminosity are extrapolated from Figure 3 in Marinucci et al. (2016) and shown here for comparison. Errors correspond to the 68 per cent confidence level for one interesting parameter. Brown stripes and violet bricks indicate a period of 5 and 28 months, respectively, not covered by observations.

rial is composed of individual spherical clouds orbiting with Keplerian velocities at a distance $R$ from the SMBH, the distance of the cloud responsible for the unveiling/eclipsing event is given by

$\mathrm{R}[\mathrm{cm}]=\frac{\mathrm{GM}_{\mathrm{BH}}(\Delta \mathrm{t})^{2} \mathrm{n}^{2}}{\mathrm{~N}_{\mathrm{H}}^{2}}$,

where $G$ is the gravitational constant, $M_{B H}$ is the BH mass, $\Delta t$ is the timescale of the column density variation, and $n$ and $N_{H}$ are the gas density and the column density of the cloud, respectively. Assuming a black hole mass $M_{B H}=10^{7}$ $\mathrm{M}_{\odot}$, as derived from water maser measurements (Greenhill et al. 1996), and a typical value for the gas density within the broad line region, such as $n=10^{10} \mathrm{~cm}^{-3}$ (Wang et al. 2012), we estimate a size for the obscuring cloud of $D \sim 150 R_{g}$ and find that the absorbers responsible for the unveiling and eclipsing event are located at a distance $R \leq(0.07 \pm 0.05) \mathrm{pc}$ and $R \leq(0.46 \pm 0.14) \mathrm{pc}$, respectively.

According to Kaspi et al. (2005), the radius of the BLR is given by

$\frac{\mathrm{R}_{\mathrm{BLR}}}{10 \mathrm{lt}-\text { days }}=0.86 \times\left(\frac{\mathrm{L}_{2-10 \mathrm{keV}}}{10^{43} \mathrm{erg} \mathrm{s}^{-1}}\right)^{0.544}$,

leading to $R_{B L R}=\left(0.014_{-0.007}^{+0.015}\right)$ pc, using our spectral fitting parameters. Moreover, using the bolometric correction from Marconi et al. (2004), we inferred a bolometric luminosity $L_{b o l}=\left(0.8_{-0.6}^{+1.2}\right) \times 10^{45} \mathrm{erg} \mathrm{s}^{-1}$, in agreement with other studies (e.g. Pier et al. 1994; Woo \& Urry 2002; Hönig et al. 2008) and leading to a dust sublimation radius $R_{d}=\left(0.36_{-0.14}^{+0.27}\right) \mathrm{pc}$, if an average dust grain size of $0.05 \mu \mathrm{m}$ with a temperature $T=1500 K$ is considered (Barvainis 1987). Assuming the torus inner walls to be at the dust sublimation radius, this value is consistent with the dimension of the dusty torus observed in infrared. Therefore, considering the gas density reported above, the obscuring clouds are constrained to be located in the innermost and hottest part of the dusty torus, or even further inside (i.e. in the BLR).

Finally, the inferred bolometric luminosity leads to a normalized accretion rate $\lambda=\frac{L_{\text {bol }}}{L_{\mathrm{Edd}}}=0.63_{-0.48}^{+0.95}$, which confirms the highly accreting nature of the source. Therefore, we cannot definitively rule out the hypothesis that at least part of the observed X-ray variability of the AGN in NGC 1068 is due to a change in the intrinsic luminosity of the central accretion disk. But, also in this latter case (i.e. Model D), a variation in $N_{H}$ is expected, even though the column density in OBS4 is unconstrained. On the other hand, if we attributed the observed X-ray variability as due only to a change in the intrinsic AGN luminosity (i.e. Model F), we obtain a scenario equivalent to that suggested by our bestfit model from a statistical point of view. However, according to Shemmer et al. (2008), the normalized accretion rate resulting in OBS4 would correspond to a power-law slope $\Gamma=1.82 \pm 0.01$, well below that observed in our analysis (i.e. $\Gamma=2.1$ ). Furthermore, the uniform $N_{H}$ - multiple $L_{X}$ scenario predicts a column density well below the average value of $10^{25} \mathrm{~cm}^{-2}$ usually observed in NGC 1068 (e.g. Bauer et al. 2015), while our assumption of a constant intrinsic luminosity of the AGN on timescales of months is supported by mid-IR and optical data. Thus, our findings firmly rule out the scenario of a single, monolithic obscuring wall, and instead support the presence of a clumpy torus surrounding the nuclear region of NGC 1068.

\section{CONCLUSIONS}

We have presented a spectral analysis of the latest $N u S$ TAR monitoring campaign of the Compton-thick Seyfert 2 galaxy NGC 1068 composed of four $\sim 50$ ks observations performed between July 2017 and February 2018 and probing 
timescales from 1 to 6 months. Our findings are summarized as follows.

- We detected one unveiling and one eclipsing event with timescales lower than 27 and 91 days, respectively, ascribed to Compton-thick material with $N_{H}=(1.8 \pm 0.8) \times 10^{24} \mathrm{~cm}^{-2}$ and $N_{H} \geq(2.4 \pm 0.5) \times 10^{24} \mathrm{~cm}^{-2}$ moving across our line of sight, allowing or preventing the intrinsic nuclear radiation from piercing through the circumnuclear matter. We can apparently locate the absorbing gas clouds to arise from the innermost part of the torus or closer, thus providing both tighter constraints on their location with respect to the previous NuSTAR monitoring campaign, and further evidence of the clumpy structure of the circumnuclear matter in this source.

- We reported a Swift-XRT detection of a transient Xray source in February 2018, which is plausibly a strongly variable ULX located at $\sim 2 \mathrm{kpc}$ from the nucleus of NGC 1068 , with a peak X-ray intrinsic luminosity of $(3.0 \pm 0.4) \times$ $10^{40} \mathrm{erg} \mathrm{s}^{-1}$ in the $2-10 \mathrm{keV}$ band.

Since its launch in June 2012, NuSTAR has observed NGC 1068 several times, sampling timescales ranging from $\sim 1$ month to $\sim 5$ years; further observations also performed simultaneously with future $\mathrm{X}$-ray observatories, like XRISM, will be certainly helpful in refining and better constraining the scenario we discussed in this paper. Furthermore, a leap in this field is expected to be achieved with the launch of the next future High-Energy X-ray Probe (HEX-P - Madsen et al. 2019) taking advantage of the complementary capability with the simultaneous ATHENA mission (Nandra et al. 2013; Barcons et al. 2017), providing the former a highenergy sensitivity with an improvement by a factor of 40 over NuSTAR in the $10-80 \mathrm{keV}$ band, and the latter a high resolution spectroscopy below $10 \mathrm{keV}$. On the other hand, different information on the nature and geometry of the circumnuclear matter in NGC 1068 can be obtained from future X-ray polarimetry missions, such as the Imaging X-ray Polarimeter Explorer (IXPE - Weisskopf et al. 2016a and 2016b, scheduled to be launched in 2021) and the enhanced X-ray Timing and Polarimetry mission ( $e X T P$ - Zhang et al. 2019 , planned to be launched in 2027). In fact, in the $2-8 \mathrm{keV}$ working band of the polarimeters on board these missions, the X-ray emission is dominated by reflection from the circumnuclear matter. A high polarization degree, dependent on the inclination and level of symmetry of the matter, is expected, with the polarization angle related to the symmetry axis. A comparison of such an axis to those of the spatially resolved inner tori and of the ionization cone will shed further light on the structure of the circumunuclear matter in NGC 1068.

\section{ACKNOWLEDGEMENTS}

We thank the anonymous referee for her/his useful comments and suggestions which improved the quality and the clarity of the paper. AZ, SB and GM acknowledge financial support from the Italian Space Agency under grant $\mathrm{n}$. 2017-14-H.O. SB acknowledges financial support from the Italian Space Agency under grant ASI-INAF I/037/12/0. $\mathrm{AM}$ and GM acknowledge financial support from the Italian Space Agency under grant ASI-INAF I/037/12/0-011/13.
FEB acknowledges support from CONICYT-Chile (Basal AFB-170002) and the Ministry of Economy, Development, and Tourism's Millennium Science Initiative through grant IC120009, awarded to the Millennium Institute of Astrophysics, MAS. CR acknowledges support from the CONICYT+PAI Convocatoria Nacional subvencion a instalacion en la academia convocatoria año 2017 PAI77170080. This research made use of data from the NuSTAR mission, a project led by the California Institute of Technology, managed by the Jet Propulsion Laboratory, and funded by NASA, and of the NuSTAR Data Analysis Software (NuSTARDAS) jointly developed by the ASI Science Data Center (ASDC, Italy) and the California Institute of Technology (Caltech, USA). We also thank the Swift team for having scheduled our ToO request as soon as possible, and provided useful data for this paper through archival XRT observations.

\section{REFERENCES}

Antonucci R., 1993, ARA\&A, 31, 473

Antonucci R. R. J. and Miller J. S., 1985, ApJ, 297, 621

Arnaud, K. A. 1996, in Astronomical Society of the Pacific Conference Series, Vol. 101, Astronomical Data Analysis Software and Systems V, G. H. Jacoby \& J. Barnes eds., p. 17

Barcons X., Barret D., Decourchelle A., den Herder J. W., Fabian A. C., Matsumoto H., Lumb D., Nandra K., Piro L., Smith R. K. and Willingale R., 2017, Astronomische Nachrichten, 338, 153

Barvainis R., 1987, ApJ, 320, 537

Bauer F. E., Arévalo P., Walton D. J., Koss M. J., Puccetti S., Gandhi P., Stern D., Alexander D. M., Baloković M., Boggs S. E., Brandt W. N., Brightman M., Christensen F. E., Comastri A., Craig W. W., Del Moro A., Hailey C. J., Harrison F. A., Hickox R., Luo B., Markwardt C. B., Marinucci A., Matt G., Rigby J. R., Rivers E., Saez C., Treister E., Urry C. M. and Zhang W. W., 2015, ApJ, 812, 116

Bianchi S., Piconcelli E., Chiaberge M., Bailón E. J., Matt G. and Fiore F., 2009, ApJ, 695, 781

Bianchi S., Maiolino R., Risaliti G., 2012, Adv. Astron, 2012, id. 782030

Bland-Hawthorn J., Gallimore J. F., Tacconi L. J., Brinks E., Baum S. A., Antonucci R. R. J. and Cecil G. N., 1997, Ap\&SS, 248, 9

Bostroem K. A., Valenti S., Sand D. J., Andrews J. E., Van Dyk S. D., Galbany L., Pooley D., Amaro R. C., Smith N., Yang S., Anupama G. C., Arcavi I., Baron E., Brown P. J., Burke J., Cartier R., Hiramatsu D., Dastidar R., Derkacy J. M., Dong Y., Egami E., Ertel S., Filippenko A. V., Fox O. D., Haislip J., Hosseinzadeh G., Howell D. A., Gangopadhyay A., Jha S. W., Kouprianov V., Kumar B., Lundquist M., Milisavljevic D., McCully C., Milne P., Misra K., Reichart D. E., Sahu D. K., Sai H., Singh A., Smith P. S., Vinko J., Wang X., Wang Y., Wheeler J. C., Williams G. G., Wyatt S., Zhang J. and Zhang X., 2019, arXiv:1909.07304

Cash W., 1976, A\&A, 52, 307

Collinge M. J. and Brandt W. N., 2000, MNRAS, 317, L35

De Cicco M., Marinucci A., Bianchi S., Piconcelli E., Violino G., Vignali C. and Nicastro F., 2015, MNRAS, 453, 2155

Elvis M., Risaliti G., Nicastro F., Miller J. M., Fiore F. and Puccetti S., 2004, ApJ, 615, L25

Fritz J., Franceschini A. and Hatziminaoglou E., 2006, MNRAS, 366,767

Gallimore J. F., Baum S. A. and O'Dea C. P., 2004, ApJ, 613, 794 
Gandhi P., Horst H., Smette A., Hönig S., Comastri A., Gilli R., Vignali C. and Duschl W., 2009, A\&A, 502, 457

García-Burillo S., Combes F., Ramos Almeida C., Usero A., Alonso-Herrero A., Hunt L. K., Rouan D., Aalto S., Querejeta M., Viti S., van der Werf P. P., Fuente A., Colina L., MartínPintado J., Henkel C., Martín S., Gratadour D., Neri R. and Tacconi L. J., 2019, arXiv:1909.00675

Gladstone J. C., Roberts T. P. and Done C., 2009, MNRAS, 397, 1836

Greenhill L. J., Gwinn C. R., Antonucci R. and Barvainis R., 1996, ApJ, 472, L21

Harrison F. A., Craig W. W., Christensen F. E., Hailey C. J., Zhang W. W. et al., 2013, ApJ, 770, 103

Hönig S. F., Prieto M. A. and Beckert T., 2008, A\&A, 485, 33

Jaffe W., Meisenheimer K., Rottgering H. J. A., Leinert Ch., Richichi A., Chesneau O., Fraix-Burnet D., GlazenborgKluttig A., Granato G.-L., Graser U., Heijligers B., Kohler R., Malbet F., Miley G.K., Paresce F., Pel J.-W., Perrin G., Przygodda F., Schoeller M., Sol H., Waters L.B.F.M., Weigelt G., Woillez J. and de Zeeuw P.T., 2004, Nature, 429, 47

Kalberla P. M. W., Burton W. B., Hartmann D., Arnal E. M., Bajaja E., Morras R. and Pöppel W. G. L., 2005, A\&A, 440, 775

Kaspi S., Maoz D., Netzer H., Peterson B. M., Vestergaard M. and Jannuzi B. T., 2005, ApJ, 62961

Imanishi M., Nakanishi K., Izumi T. and Wada K., 2018, ApJ, 853, L25

Impellizzeri C. M. V., Gallimore J. F., Baum S. A., Elitzur M., Davies R., Lutz D., Maiolino R., Marconi A., Nikutta R., O'Dea C. P. and Sani E., 2019, arXiv:1908.07981

Lamastra A., Bianchi S., Matt G., Perola G. C., Barcons X. and Carrera F. J., 2009, A\&A, 504, 73

López-Gonzaga N., Asmus D., Bauer F. E., Tristram K. R. W. Burtscher L., Marinucci A., Matt G. and Harrison F. A., 2017, A\&A, 602, A78

Lynden-Bell D., 1969, Nature, 223, 690

Madsen K. K., Alexander D., Bachetti M., Ballantyne D., Baloković M., Boggs S., Boorman P., Brandt N. W., Brenneman L., Christensen F., Civano F., Connors R., Descalle M., Elvis M., Fabian A., Ferreira D., Fornasini F., Fuerst F., García J., Gellert N., Grefenstette B., Grindlay J., Heida M., Hickox R., Miyasaka H., Hornschmeier A., Hornstrup A., Jaodand A., Kamraj N., Krawczynski H., Lansbury G., Lohfink A., Matt G., Middleton M., Miller J., Pottschmidt K., Ricci C., Stern D., Vogel J., Walton D., Wilms J., Wik D., Windt D. and Zhang W., 2019, Astro2020: Decadal Survey on Astronomy and Astrophysics, APC white papers, no. 166; Bulletin of the American Astronomical Society, Vol. 51, Issue 7, id. 166

Marconi A., Risaliti G., Gilli R. Hunt L. K., Maiolino R. and Salvati M., 2004, MNRAS, 351, 169

Marinucci A., Bianchi S., Matt G., Alexander D. M., Baloković M., Bauer F. E., Brandt W. N., Gandhi P., Guainazzi M., Harrison F. A., Iwasawa K., Koss M., Madsen K. K., Nicastro F., Puccetti S., Ricci C., Stern D. and Walton D. J., 2016, MNRAS, 456, L94

Middleton M. J., Sutton A. D., Roberts T. P., Jackson F. E. and Done C., 2012, MNRAS, 420, 2969

Middleton M. J., Miller-Jones J. C. A., Markoff S., Fender R., Henze M., Hurley-Walker N., Scaife A. M. M., Roberts T. P., Walton D. J., Carpenter J., Macquart J.-P., Bower G. C., Gurwell M., Pietsch W., Haberl F., Harris J., Daniel M., Miah J., Done C., Morgan J. S., Dickinson H., Charles P., Burwitz V., Della Valle M., Freyberg M., Greiner J., Hernanz M., Hartmann D. H., Hatzidimitriou D., Riffeser A., Sala G., Seitz S., Reig P., Rau A., Orio M., Titterington D. and Grainge K., 2013, Nature, 493, 187

Miniutti G., Ponti G., Dadina M., Cappi M., Malaguti G., Fabian A. C. and Gandhi P., 2006, MNRAS, 373, L1
Murphy K. D. and Yaqoob T., 2009, MNRAS, 397, 1549

Nandra K., Barret D., Barcons X., Fabian A., den Herder J.-W. et al., 2013, arXiv:1306.2307

Nenkova M., Sirocky M. M., Nikutta R., Ivezić Z. and Elitzur M., 2008a, ApJ, 685, 147

Nenkova M., Sirocky M. M., Nikutta R., Ivezić Z. and Elitzur M., 2008b, ApJ, 685, 160

Netzer H., 2015, ARAA, 53, 365

Piconcelli E., Bianchi S., Vignali C., Jiménez-Bailón E. and Fiore F., A\&A, 534, A126

Pier E. A. and Krolik J. H., 1992, ApJ, 401, 99

Pier E. A. and Krolik J. H., 1993, ApJ, 418, 673

Pier E. A., Antonucci R., Hurt T., Kriss G. and Krolik J., 1994, ApJ, 428, 124

Pintore F., Zampieri L., Stella L., Wolter A., Mereghetti S. and Israel G. L., 2017, ApJ, 836, 113

Pintore F., Belfiore A., Novara G., Salvaterra R., Marelli M., De Luca A., Rigoselli M., Israel G., Rodriguez G., Mereghetti S., Wolter A., Walton D. J., Fuerst F., Ambrosi E., Zampieri L., Tiengo A. and Salvaggio C., 2018, MNRAS, 477, L90

Puccetti S., Fiore F., Risaliti G., Capalbi M., Elvis M. and Nicastro F., 2007, MNRAS, 377, 607

Raban D., Jaffe W., Röttgering H., Meisenheimer K. and Tristram, K. R. W., 2009, MNRAS, 394, 1325

Ramos Almeida C. and Ricci C., 2017, Nature Astronomy, 1, 679

Risaliti G., Elvis M. and Nicastro F., 2002, ApJ, 571, 234

Risaliti G., Elvis M., Fabbiano G., Baldi A. and Zezas A., 2005, ApJ, 623, L93

Rivers E., Risaliti G., Walton D. J., Harrison F., Arévalo P., Bauer F. E., Boggs S. E., Brenneman L. W., Brightman M., Christensen F. E., Craig W. W., Furst F., Hailey C. J., Hickox R. C., Marinucci A., Reeves J., Stern D. and Zhang W. W., 2015a, ApJ, 804, 107

Rivers E., Baloković M., Arévalo P., Bauer F. E., Boggs S. E., Brandt W. N., Brightman M., Christensen F. E., Craig W. W., Gandhi P., Hailey C. J., Harrison F., Koss M., Ricci C., Stern D., Walton D. J. and Zhang W. W., 2015b, ApJ, 815, 55

Shemmer O., Brandt W. N., Netzer H., Maiolino R. and Kaspi S., 2008, ApJ, 682, 81

Smith D. A. \& Wilson A. S., 2003, ApJ, 591, 138

Stobbart A. -M., Roberts T. P. and Wilms J., 2006, MNRAS, 368,397

Telesco C. M. and Decher R., 1988, ApJ, 334, 573

Tully R. B., 1988, Nearby Galaxies Catalog, Cambridge and New York, Cambridge Univ. Press

Verner D. A., Ferland G. J., Korista K. T. and Yakovlev D. G., 1996, ApJ, 465, 487

Walton D. J., Harrison F. A., Bachetti M., Barret D., Boggs S. E., Christensen F. E., Craig W. W., Fuerst F., Grefenstette B. W., Hailey C. J., Madsen K. K., Middleton M. J., Rana V., Roberts T. P., Stern D., Sutton A. D., Webb N. and Zhang W., 2015, ApJ, 799, 122

Walton D. J., Fürst F., Heida M., Harrison F. A., Barret D., Stern D., Bachetti M., Brightman M., Fabian A. C. and Middleton M. J., 2018, ApJ, 856, 128

Wang Y., Ferland G. J., Hu C., Wang J.-M. and Du P., 2012, MNRAS, 424, 2255

Weisskopf M. C., Ramsey B., O'Dell S. L., Tennant A., Elsner R., Soffitta P., Bellazzini R., Costa E., Kolodziejczak J., Kaspi V., Muleri F., Marshall H., Matt G. and Romani R., 2016a, Proc. SPIE 9905, Space Telescopes and Instrumentation 2016: Ultraviolet to Gamma Ray, 990517

Weisskopf M. C., Ramsey B., O'Dell S. L., Tennant A., Elsner R., Soffitta P., Bellazzini R., Costa E., Kolodziejczak J., Kaspi V., Muleri F., Marshall H., Matt G. and Romani R., 2016b, Results in Physics, 6, 1179

Wilms J., Allen A. and McCray R., 2000, ApJ, 542, 914 
Wittkowski M., Kervella P., Arsenault R., Paresce F., Beckert T. and Weigelt G., 2004, A\&A, 418, L39

Woo J.-H. and Urry C. M., 2002, ApJ, 579, 530

Yang G., Brandt W. N., Luo B., Xue Y. Q., Bauer F. E., Sun M. Y., Kim S., Schulze S., Zheng X. C., Paolillo M., Shemmer O., Liu T., Schneider D. P., Vignali C., Vito F. and Wang J. -X., 2016, ApJ, 831, 145

Yaqoob T., 2012, MNRAS, 423, 3360

Yaqoob T., Tatum M. M., Scholtes A., Gottlieb A. and Turner T. J., 2015, MNRAS, 454, 973

Zhang S.-N., Santangelo A., Feroci M., Xu Y., Lu F. et al., 2019, Science China Physics, Mechanics \& Astronomy, 62, 29502

Zhao X., Marchesi S., Ajello M., Marcotulli L., Cusumano G., La Parola V. and Vignali C., 2019, ApJ, 870, 60

\section{APPENDIX A: A NEW ULX DETECTION IN NGC 1068?}

NGC 1068 is not only one of the best known Seyfert 2 galaxies in the local universe, but also a powerful starburst galaxy with a star-formation rate $\dot{M}>36 M_{\odot} y r^{-1}$ (i.e. at least a factor of 7 greater than the SFR in our Galaxy). In particular, infrared observations indicate that the star formation in this galaxy is primarily distributed within the central $\sim 3 \mathrm{kpc}$ in two very extended complexes, one located to the north and the other to the southwest of the nucleus (Telesco \& Decher 1988).

The NuSTAR images taken in February 2018 in the 3-5.5 keV energy band suggest the presence of a transient object at a distance of $\sim 30$ arcsec from the nuclear region (see Figure A1). Analysis of the simultaneous Swift-XRT image confirms this hypothesis (see Figure A2), allowing us to ascribe the 3-5.5 keV excess observed in February 2018 to the appearance of a flaring source at a distance of $\sim 2 \mathrm{kpc}$ from the nucleus of NGC 1068 (at the distance of NGC 1068 $1 "=72$ pc - Bland-Hawthorn et al. 1997).

To identify this flaring source, we overlaid the five point-like sources previously detected with Chandra (Smith \& Wilson 2003), within 20 arcsec of its position, on the Swift-XRT 3-5.5 keV image (see Figure A2). Among these point-like objects, the brightest one (with more than 50 counts in the Chandra band and with a SNR $>7$ ) is CXOU J024244.0-000035, with a reported 0.4-8 keV luminosity $L_{0.4-8}=6.8 \times 10^{38} \mathrm{erg} \mathrm{s}^{-1}$, corresponding to a $2-10 \mathrm{keV}$ luminosity $L_{2-10}=4.8 \times 10^{38} \mathrm{erg} \mathrm{s}^{-1}$, which is almost two orders of magnitude lower than the one observed during our monitoring. No known X-ray sources appear to lie closer than $d \simeq 8$ arcsec from the ULX centroid in the Swift-XRT image, neither the supernova exploded in NGC 1068 in November 2018 (Bostroem et al. 2019) matches the location of the ULX; therefore, we conclude that there is no robust identification with previously detected sources.

To obtain NuSTAR FPMA and FPMB spectra of the ULX, we computed difference spectra between the ones extracted from the February 2018 and November 2017 observations, already described in Section 2.1. Then, we rebinned the two spectra in order to have a SNR greater than $2 \sigma$ in each spectral channel. With this requirement, no detected spectral bins are present above $16 \mathrm{keV}$ and $12 \mathrm{keV}$ for FPMA and FPMB, respectively.

We used the C-statistic to simultaneously fit the XRT, FPMA and FPMB spectra obtained in February 2018. An
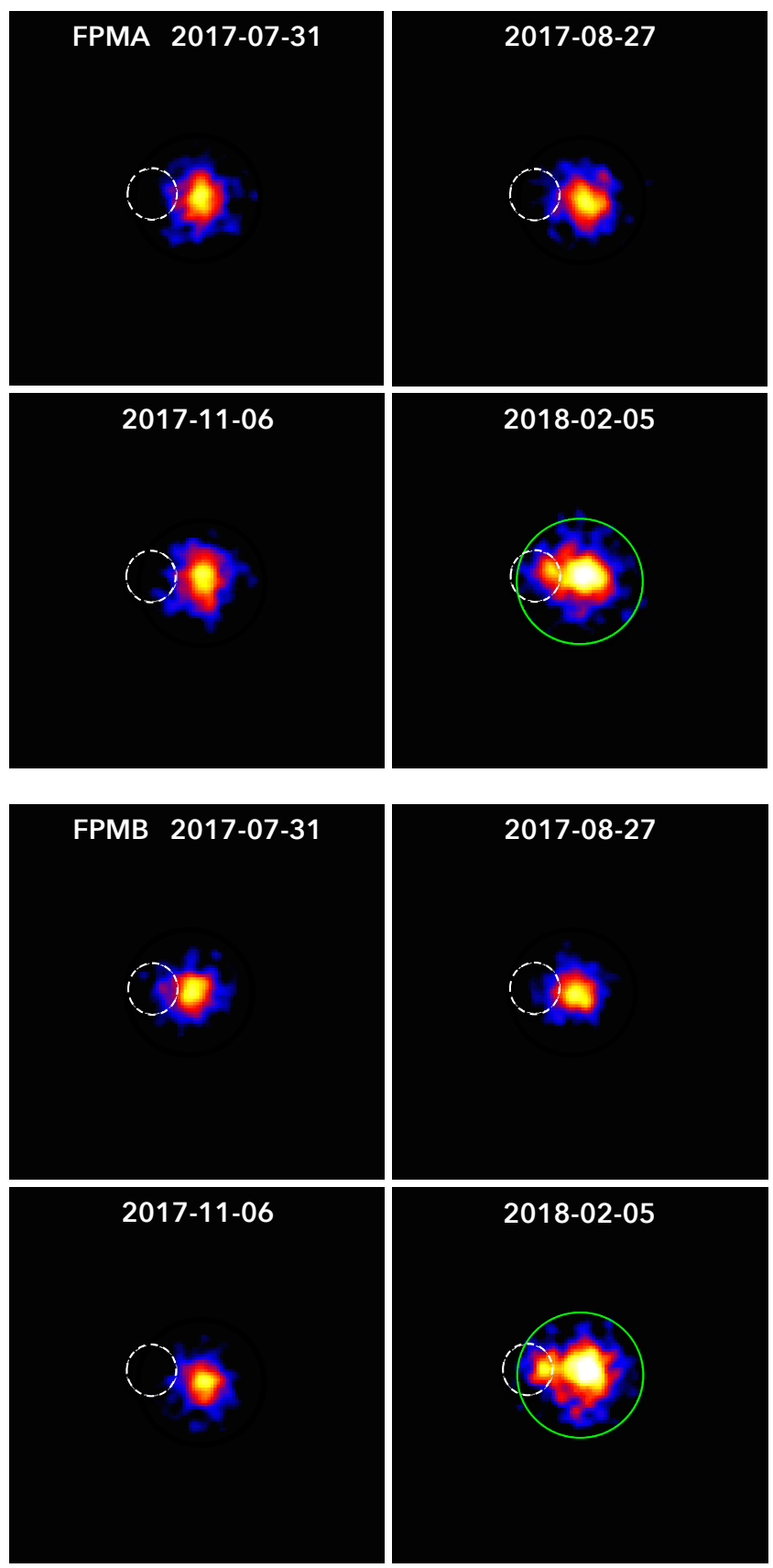

Figure A1. NuSTAR FPMA (top panels) and FPMB (bottom panels) images of the central 5' $\times 5$ ' regions in the $3-5.5 \mathrm{keV}$ band smoothed with a 3 pixels radius Gaussian filter. The circular region with a radius of 50 arcsec used for extracting the source spectra is shown in OBS4 (green solid circle), while the 20 arcsec circular region used to extracted the Swift-XRT spectrum is overplotted in all panels (white dotted circles).

absorbed cut-off power-law provides an acceptable description of the data, with a fixed value for the Galactic column density $\left(N_{H}^{G a l}=3 \times 10^{20} \mathrm{~cm}^{-2}-\right.$ Kalberla et al. 2005), a photon index $\Gamma=1.5 \pm 0.4$, a cut-off energy $E_{c} \geq 5 \mathrm{keV}$ and a normalization $N=\left(2.6_{-0.6}^{+0.7}\right) \times 10^{-4} \mathrm{ph} \mathrm{cm}^{-2} \mathrm{~s}^{-1} \mathrm{keV}^{-1}$. We retrieved a $C / d o f=43.3 / 45$ and our best fit is shown in Figure A3. The observed flux in the $2-10 \mathrm{keV}$ band is 


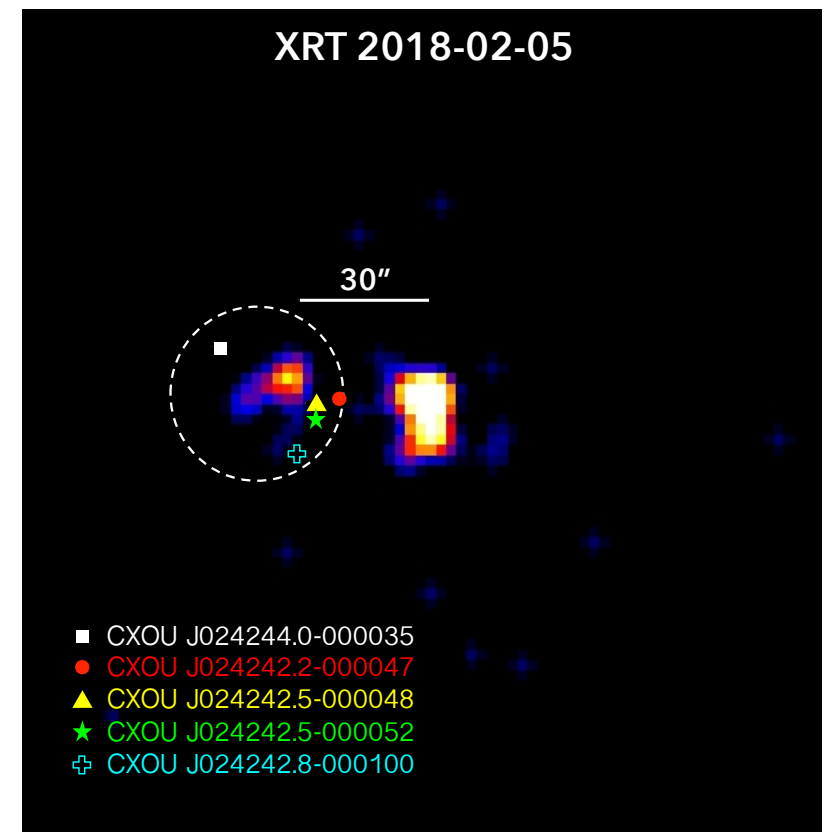

Figure A2. Swift-XRT image of the central 3' $\times 3$ ' region, obtained in February 2018, in the 3-5.5 keV band. The dashed white circular region with a radius of 20 arcsec used for extracting the ULX spectrum is shown. We over-imposed the five different pointlike sources previously detected with Chandra and located within a 20 arcsec radius region from the ULX, from Smith \& Wilson (2003). The ULX is not detected in November 2017 nor in June 2018.

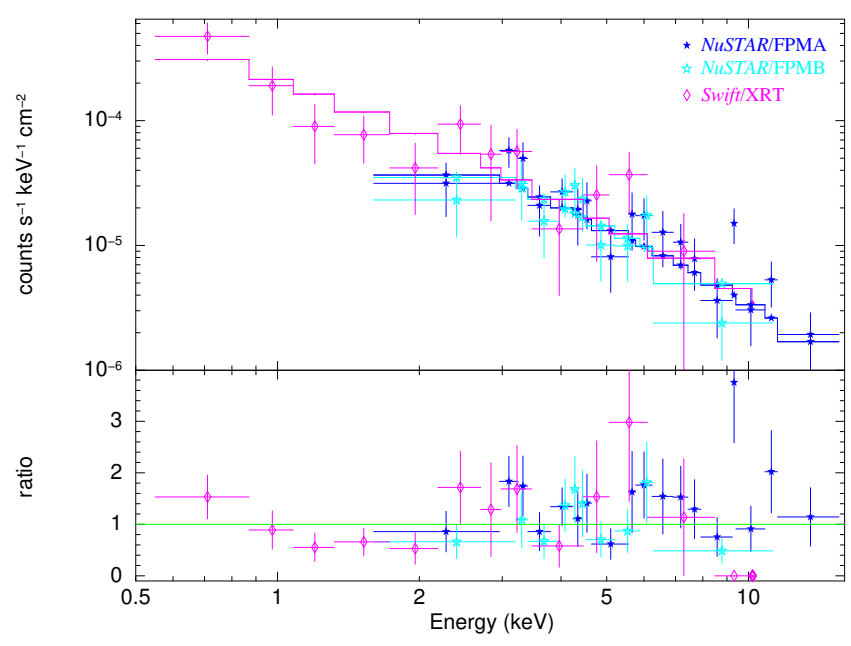

Figure A3. Top panel. XRT (magenta diamonds), FPMA (blue filled stars) and FPMB (cyan empty stars) ULX spectra taken in February 2018. Bottom panel. Data/model ratio with respect to a model (solid green line) composed of an absorbed power-law with a photon index $\Gamma=1.5 \pm 0.4$ and a cut-off energy $E_{c} \geq 5 \mathrm{keV}$.

$F_{2-10}=(9.4 \pm 1.0) \times 10^{-13} \mathrm{erg} \mathrm{cm}^{-2} \mathrm{~s}^{-1}$, corresponding to an intrinsic luminosity $L_{2-10}=(3.0 \pm 0.4) \times 10^{40} \mathrm{erg} \mathrm{s}^{-1}$ at the distance of NGC 1068 .

Since the source was not detected before, we requested and obtained a $1.5 \mathrm{ks}$ ToO observation with Swift-XRT on June 15, 2018 (NGC 1068 was not observable before this date due to Sun occultation) to confirm the presence of this new source. Unfortunately, the transient source was no longer visible in the Swift-XRT image in the $3-5.5 \mathrm{keV}$ band, and the spectrum extracted from a circular region of 20 arcsec centered on the position of the ULX, as observed in February 2018, showed a dramatic drop of the count-rate in the $2-10 \mathrm{keV}$ band (i.e. about one order of magnitude, from $0.010 \pm 0.002$ counts/s in February 2018 to $0.0013 \pm 0.0009$ counts/s in June 2018). This prevented further detailed study of this transient source, allowing us to determine only a variability timescale less than $\sim 3$ months. However, we note that transient ULXs are already known (e.g. Middleton et al. 2012, 2013; Pintore et al. 2018), and that even high luminosity ULXs can vary on very short timescales, such as ULX1 in NGC 5907 (Walton et al. 2015).

\section{APPENDIX B: THE BAUER MODEL}

In 2015, Bauer et al. (2015) characterized the multi-epoch X-ray spectra of NGC 1068 analyzing high-quality observations performed from 1996 until 2012 by different X-ray observatories. In particular, NuSTAR and XMM-Newton data were used to study in detail the total emission of the source, while the higher spectral and angular resolution of Chan$d r a$ (HETG and ACIS, respectively) enabled the authors to remove potential host-galaxy contamination, spatially separating the nuclear spectrum from diffuse and off-nuclear point-source emission at least below $8 \mathrm{keV}$. Swift, Suzaku and BeppoSAX observations were used for points of comparison.

The broadband emission of NGC 1068 was modeled with a combination of a heavily Compton-thick transmitted power law, scattering by both warm and cold reflectors, radiative recombination continuum (RRC) and line emission, and off-nuclear point-source emission (see Figure 12 in Bauer et al. 2015 for the fractional contributions of the different spectral components). No angular dependence of the nuclear emission spectral shape is assumed (i.e. all scattering component have the same photon index), nor is relativistic reflection from the accretion disk considered, due to the inclination of the source and the dominance of scattering and absorption from cold distant material.

To construct a robust model for the nuclear X-ray spectrum of NGC 1068, both the point-like nuclear emission and the diffuse emission and point source contamination from the host galaxy must be taken into account. The extended emission can be effectively constrained below $8 \mathrm{keV}$ by Chandra imaging, leaving considerable degeneracy at higher energies. Since the aim is to provide the best constraints on the properties of the reflectors, data below $2 \mathrm{keV}$ are not considered, and all the spectral components that are well-constrained through a separate fit of the nuclear and host galaxy contribution (i.e. extranuclear point sources, RRC and line emission) are fixed during the combined fit (see Bauer et al. 2015 for the adopted values of each spectral component).

The AGN intrinsic continuum is well-described by a highly absorbed $\left(N_{H}=10^{25} \mathrm{~cm}^{-2}\right)$ power-law with a photon index $\Gamma=2.10_{-0.07}^{+0.06}$ and an energy cut-off $E_{c}=128_{-44}^{+115}$ $\mathrm{keV}$, while a multi-component reflector with three distinct column densities $\left(N_{H, 1} \sim 10^{25} \mathrm{~cm}^{-2}, N_{H, 2}=(1.4 \pm 0.1) \times 10^{23}\right.$ $\left.\mathrm{cm}^{-2}, N_{H, 3}=\left(5.0_{-1.9}^{+4.2}\right) \times 10^{24} \mathrm{~cm}^{-2}\right)$ reproduces the complex structure of the circumnuclear matter (i.e. Model M2d in 


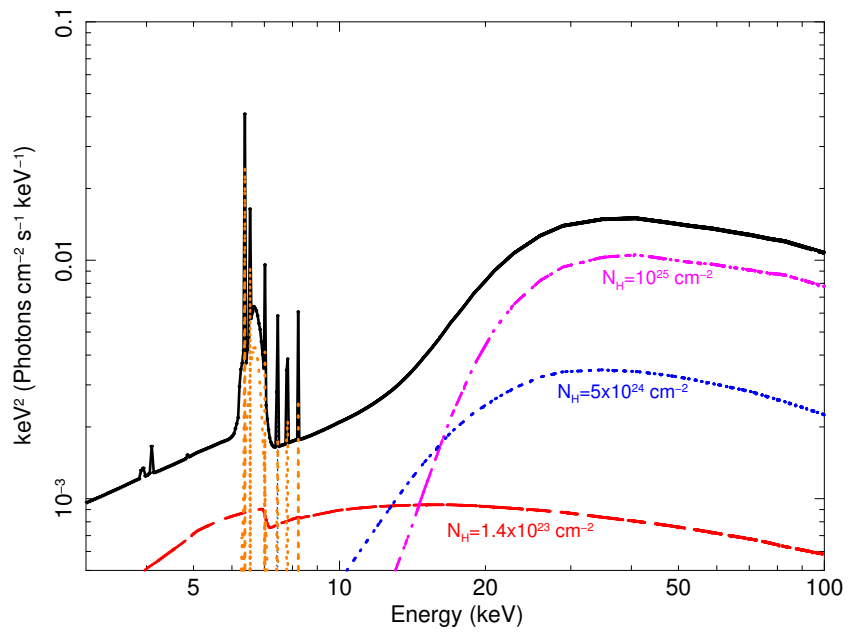

Figure B1. Theoretical best-fit model of NGC 1068 adopted by Bauer et al. 2015 (solid black line). Emission lines (orange dotted lines in the 6-9 keV energy range), nuclear (dashed red and dot-dashed magenta lines) and more distant (blue dotted line) reflectors represent the reflection components arising from different column densities, while the nuclear intrinsic emission is completely absorbed by heavily Compton-thick matter.

Bauer et al. 2015 - see Figure B1). The spectral features attributed to the $N_{H, 1}$ and $N_{H, 2}$ components arise from the central region, within 2 arcsec from the nucleus, while $N_{H, 3}$ corresponds to regions outside the central 2 arcsec. In particular, the higher $N_{H}$ component provides the bulk of the flux to the Compton hump, while the lower $N_{H}$ component contributes primarily to the iron line emission and reproduces the curvature of the continuum around $10 \mathrm{keV}$, effectively decoupling the two key features of Compton reflection. The inclination angles of the two nuclear scatterers with respect to the line of sight are $90^{\circ}$ and $0^{\circ}$, respectively, in order to reproduce a clumpy torus distribution with the edge-on scatterer accounting for the photons reprocessed by the obscuring material lying between the AGN and the observer, while the face-on scatterer mimics the reprocessed emission coming from back-side reflection. These latter photons have a smaller chance of being absorbed before reaching the observer, making the MYTORUS $0^{\circ}$ component relevant in a patchy torus scenario (Yaqoob et al. 2015; Zhao et al. 2019). On the other hand, the third reflector on more extended scales $(>140 \mathrm{pc})$ with an inclination of $0^{\circ}$ provides almost $30 \%$ of the neutral iron $\mathrm{K} \alpha$ line flux and could represent material within the ionization cones.

\section{APPENDIX C: CALIBRATION ISSUES}

As reported in Section 3, when modelling the NuSTAR Xray spectra of NGC 1068, we obtained significant residuals at $\sim 6 \mathrm{keV}$ (see panel (a) in Figure 3 ). Comparing our monitoring observations with older data obtained during the previous NuSTAR monitoring campaign in 2014-2015, we find lack of temporal dependence of the iron line profile (see Figure C1). Assuming that the slight variations of the blue data are due to the appearence of the ULX in February 2018 (see Appendix A), the line profiles are sufficiently similar to each
Table C1. Comparison of the $6 \mathrm{keV}$ line component between old and new NuSTAR observations.

\begin{tabular}{lll}
\hline & FPMA $^{\dagger}$ & FPMB $^{\dagger}$ \\
\hline Aug 2014 & $1.8 \pm 0.5$ & $1.8 \pm 0.5$ \\
Feb 2015 & $2.0 \pm 0.4$ & $2.0 \pm 0.5$ \\
OBS1 (Jul 2017) & $1.7 \pm 0.5$ & $2.3 \pm 0.5$ \\
OBS2 (Aug 2017) & $1.3 \pm 0.5$ & $2.4 \pm 0.5$ \\
OBS3 (Nov 2017) & $2.0 \pm 0.5$ & $2.1 \pm 0.5$ \\
OBS4 (Feb 2018) & $2.1 \pm 0.5$ & $2.1 \pm 0.5$ \\
\hline
\end{tabular}

Note. ${ }^{\dagger}$ Normalization of the spurious line component, in units of $10^{-5} \mathrm{ph} \mathrm{cm}^{-2} \mathrm{~s}^{-1} \mathrm{keV}^{-1}$, fitting the data with Model E.

Table C2. Energy and normalization of the $6 \mathrm{keV}$ line component in the simultaneous XMM-Newton and NuSTAR observations performed in August 2014. Both spectra are fitted with a phenomenological model.

\begin{tabular}{lcc}
\hline & $\mathrm{E}(\mathrm{keV})$ & norm $\left(\mathrm{ph} \mathrm{cm}^{-2} \mathrm{~s}^{-1} \mathrm{keV}^{-1}\right)$ \\
\hline XMM-Newton & $6.0^{\dagger}$ & $\leq 1.9 \times 10^{-6}$ \\
NuSTAR/FPMA & $6.0 \pm 0.1$ & $(1.4 \pm 0.5) \times 10^{-5}$ \\
NuSTAR/FPMB & $6.1 \pm 0.1$ & $(2.5 \pm 0.6) \times 10^{-5}$ \\
\hline
\end{tabular}

Note. ${ }^{\dagger}$ Fixed value.

other to conclude that their variance is purely statistical. We note that no issues were reported in Marinucci et al. (2016) because they only considered NUSTAR data above $8 \mathrm{keV}$, using simultaneous $X M M-N e w t o n$ data at lower energies.

Fitting the residuals at $\sim 6 \mathrm{keV}$ with a further emission line (i.e. a gauss component in XSPEC) with respect to Model A, we obtain energies and normalization values fully consistent with each other, both in FPMA and FPMB (see Table C1). We note that for the data reduction of the older NuSTAR observations we used the same procedure explained in Section 2.1, binning the data using OBS2 as a template (i.e. the August 2014 and February 2015 spectra have the same energy bins of the August 2017 one).

As a further step, we take advantage of the simultaneous XMM-Newton and NuSTAR observations performed in August 2014 (OBSID 0740060401 and 60302003004, respectively), to evaluate the possible presence of a line at $\sim 6 \mathrm{keV}$ in data with higher spectral resolution. The $X M M-$ Newton data were reduced using the latest CCF and the pn spectrum was extracted from a circular region with a 40 arcsec radius centered on the source, and binned in order to oversample the instrumental resolution by at least a factor of 3 and to have no less than 30 counts in each background-subtracted spectral channel. We note that the extraction region for the source spectrum is smaller than those used to extract the $N u S T A R$ spectra, but no sources of emission were present beyond 40" within the EPIC images. Fitting both XMM-Newton and NuSTAR observations with a phenomenological model (i.e. zpow+pexrav+several zgauss components in XSPEC), we obtain the best fit in Figure C2. XMM-Newton data are well reproduced by the model, while significant residuals at $\sim 6 \mathrm{keV}$ are clearly visible in NuSTAR spectra (see red and green spectral bins in panel (a)). To reproduce these residuals, an additional gauss component is needed. It is worth noting that the upper limit to the flux of an emission line at that energy in the $X M M$ Newton spectrum is much lower and inconsistent with the measurement in NuSTAR (see Table C2).

All the previous findings suggest that the significant 

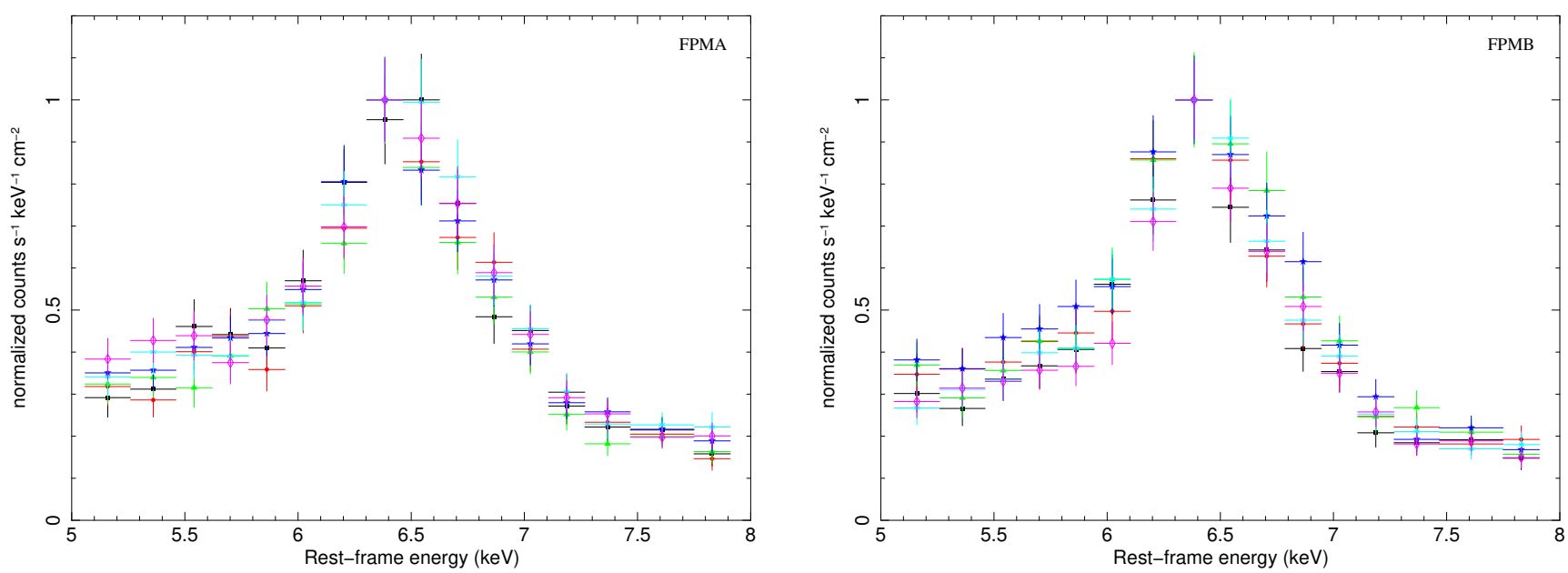

Figure C1. FPMA (left panel) and FPMB (right panel) spectra of NGC 1068 in the 5-8 keV range, normalizing to one the intensity of the highest channel. Black squares, red circles, green triangles, blue stars, cyan asterisks and magenta diamonds represent OBS1, OBS2, OBS3, OBS4, August 2014 and February 2015 observations, respectively. For clarity purposes, all the observations have the same energy bins.

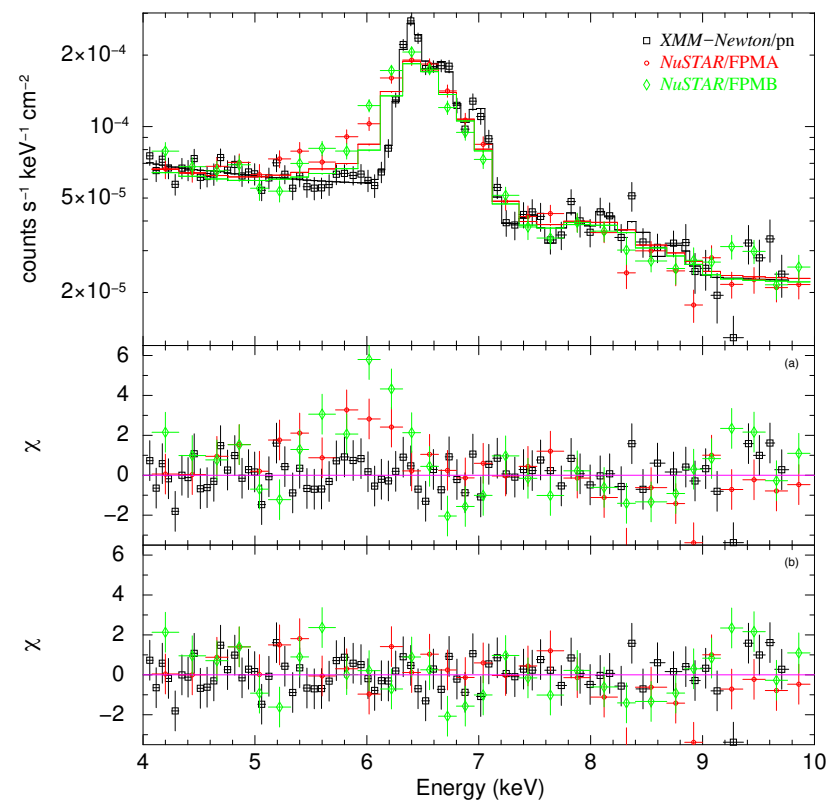

Figure C2. Upper panel. XMM-Newton/pn (black squares) and NuSTAR/FPMA (red circles) and FPMB (green diamonds) spectra of NGC 1068 performed simultaneously in August 2014, showing the 4-10 keV energy range. Lower panels. Residuals plotted in terms of sigmas with respect to the phenomenological model (panel (a)) and fitting the NuSTAR spectra with an additional emission line (panel (b)). We refer to the text for further details.

residuals observed in Model A have no astrophysical origin, but are probably due to calibration issues; therefore, to account for them, we added a spurious emission line (modeled in XSPEC with a gauss component with $\sigma=0$ ) in our bestfit model (Model E), leaving both its energy and flux free to vary between both the observations and the two NuSTAR focal planes.
An analogous feature is also observed in ESO 138-G1 (Zappacosta et al. in prep.), which is another Compton-thick AGN with a very large equivalent width of the $\mathrm{Fe} \mathrm{K} \alpha$ line (e.g. Collinge \& Brandt 2000, Piconcelli et al. 2011, De Cicco et al. 2015).

This paper has been typeset from a $\mathrm{T}_{\mathrm{E}} \mathrm{X} / \mathrm{LAT} \mathrm{T} \mathrm{X}$ file prepared by the author. 